IMA Journal of Applied Mathematics (2007) 72, 302-330

doi:10.1093/imamat/hxm005

Advance Access publication on March 22, 2007

\title{
Mathematical modelling of the catalyst layer of a polymer electrolyte fuel cell
}

\author{
A. A. SHAH $\dagger$ \\ Department of Mathematics, Simon Fraser University, 8888 University Drive, Burnaby, \\ British Columbia, Canada V5A 1 S6 \\ GWANG-SOO KIM \\ Ballard Power Systems, 4343 North Fraser Way, Burnaby, British Columbia, Canada V5J 5J9 \\ AND \\ K. PROMisLOW \\ Department of Mathematics, Michigan State University, East Lansing MI 48824, USA
}

[Received on 5 December 2005; accepted on 19 November 2006]

\begin{abstract}
In this paper, we derive a mathematical model for the cathode catalyst layer of a polymer electrolyte fuel cell. The model explicitly incorporates the restriction placed on oxygen in reaching the reaction sites, capturing the experimentally observed fall in the current density to a limiting value at low cell voltages. Temperature variations and interfacial transfer of $\mathrm{O}_{2}$ between the dissolved and gas phases are also included. Bounds on the solutions are derived from which we provide a rigourous proof that the model admits a solution. Of particular interest are the maximum and minimum attainable values. We perform an asymptotic analysis in several limits inherent in the problem by identifying important groupings of parameters. This analysis reveals a number of key relationships between the solutions, including the current density, and the composition of the layer. A comparison of numerically computed solutions and asymptotic solutions shows very good agreement. Implications of the results are discussed and future work is outlined.
\end{abstract}

Keywords: fuel cell; catalyst layer; oxygen diffusion; temperature variations; bounds; asymptotic analysis.

\section{Introduction}

The polymer electrolyte (or proton exchange membrane) fuel cell (PEMFC) is a promising energy source, particularly for application in the automotive industry. Its high power density and low operating temperature make it an attractive, environmentally friendly alternative to methods based on hydrocarbon fuels. Before the benefits of this technology can be realized, several important practical issues need to be resolved with the overarching aims of reducing the manufacturing cost and optimizing the performance and durability. The heart of a single PEMFC (see Fig. 1) is composed of a proton conducting membrane (typically Nafion) sandwiched between an anode and a cathode. The latter are formed from sheets of carbon paper and are commonly referred to as gas diffusion layers (GDL). Catalyst layers, composed roughly of carbon-supported platinum $(\mathrm{Pt})$ particles and an electrolyte (sometimes called ionomer), are

\footnotetext{
$\dagger$ Present address: School of Engineering, University of Southampton, SO17 1BJ Southampton, UK. Email: ashah@pims. math.ca, a.shah@soton.ac.uk
}

(C) The Author 2007. Published by Oxford University Press on behalf of the Institute of Mathematics and its Applications. All rights reserved. 


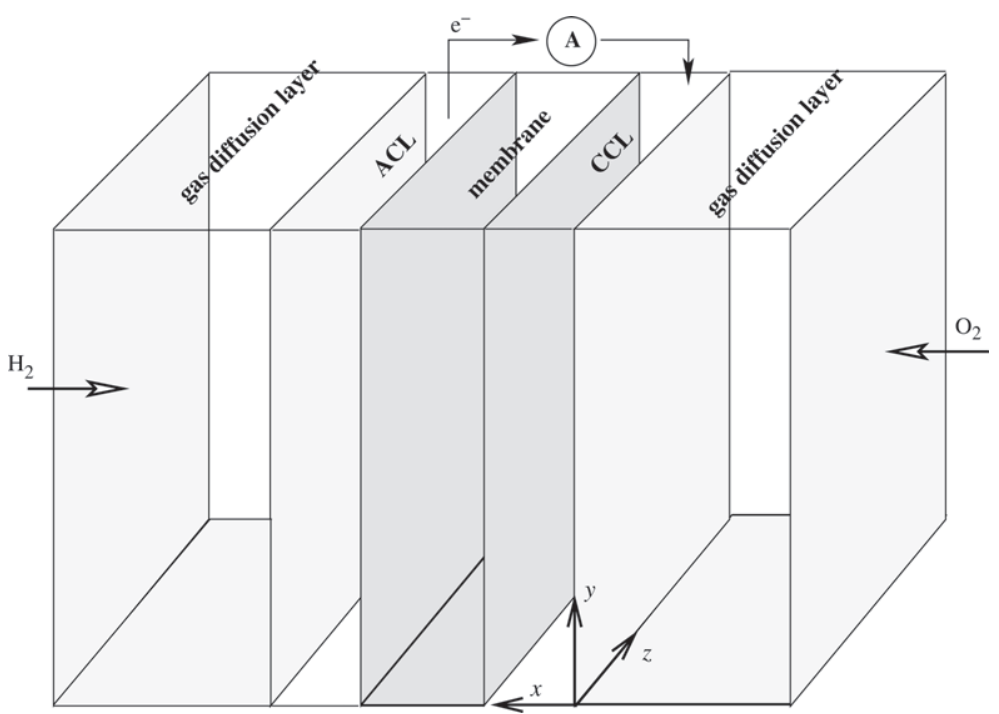

FIG. 1. A schematic of a single polymer electrolyte fuel cell. Not shown are the gas channels through which the reactants are fed. They feature in the model through the boundary conditions prescribed at the interfaces between the CCL/membrane and CCL/GDL.

pressed between the electrodes and the membrane. The basic principle of the PEMFC is to convert chemical energy into electrical energy. At the anode, hydrogen $\left(\mathrm{H}_{2}\right)$ is fed to multiple gas channels and delivered to the anode catalyst layer through the GDL. Electro-oxidation of the $\mathrm{H}_{2}$ adsorbed on the $\mathrm{Pt}$ surfaces forms protons $\left(\mathrm{p}^{+}\right)$and electrons $\left(\mathrm{e}^{-}\right)$; $\mathrm{e}^{-}$migrate through an external circuit and $\mathrm{p}^{+}$through the membrane. Oxygen $\left(\mathrm{O}_{2}\right)$ is similarly fed at the cathode and combines with $\mathrm{p}^{+}$and $\mathrm{e}^{-}$in the cathode catalyst layer (CCL) to form water. The Pt (or Pt alloy) catalyst is necessary; without it, the reactions are too weak to support a meaningful current. The cost of Pt immediately presents the challenge of optimizing its use. The required amount of Pt has seen a 10-fold reduction in the last few years, but the percentage that is 'utilized' is still very low (approximately 20\%).

The catalyst layers are arguably the most complex components of the fuel cell. The gaseous species and liquid water flow through the pores formed between the solid components (carbon, Pt and electrolyte). The reactants and water also exist as species dissolved in the electrolyte. $\mathrm{e}^{-}$migrate through the matrix of carbon grains and $\mathrm{p}^{+}$through tortuous pathways provided by the electrolyte. Of the two catalyst layers, the CCL receives most attention, primarily because of the relatively slow rate of $\mathrm{O}_{2}$ reduction reaction (ORR) and because the management of water (produced in the CCL) is an issue of great practical importance; although liquid water can hamper the transport of gaseous $\mathrm{O}_{2}$, the conductivity of the membrane (and electrolyte) increases with increased water content.

An effective tool for measuring the performance of a PEMFC is the polarization curve, a plot of cell voltage (measuring the potential drop from the anode to the cathode) against current density. A typical such curve is sketched in Fig. 2, from which we can identify three primary sources of voltage loss:

- The activation losses relate to the sluggish nature of the reactions at the electrodes, particularly of the adsorption step of the ORR at the cathode.

- The ohmic losses result mainly from ionic and electronic resistances, but contain a contribution from the limitation placed on the $\mathrm{O}_{2}$ in reaching the Pt surfaces in the CCL. 


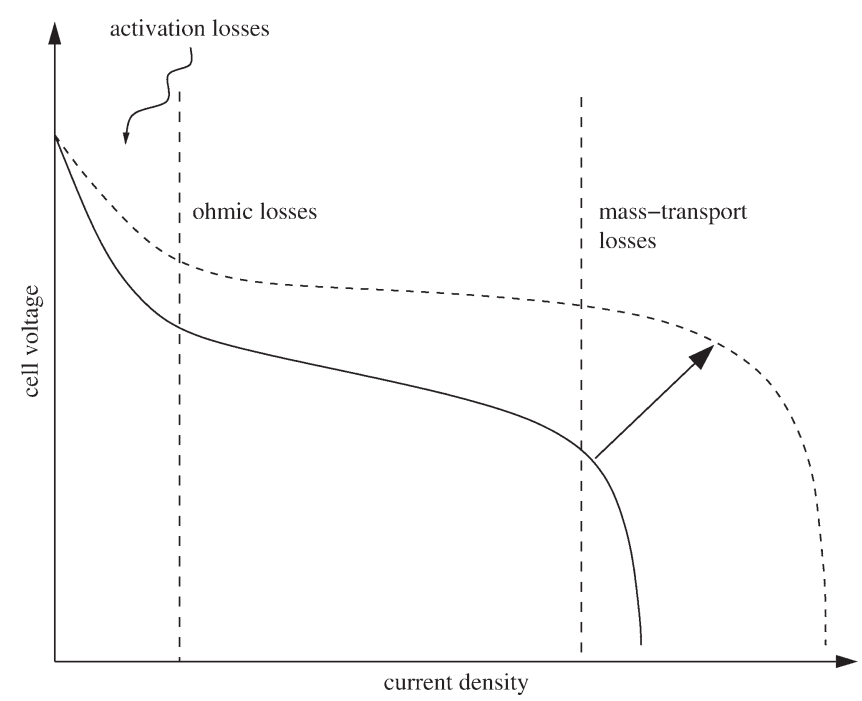

FIG. 2. The typical profile of a polarization curve exhibiting all three forms of voltage loss.

- The mass-transport losses arise almost entirely from the $\mathrm{O}_{2}$-transport limitation just described. Their effect on performance is a sharp reduction in the current density to a limiting value at low cell voltage.

Improved performance (increased total power and higher current) translates effectively to an increased limiting current density and a reduction in the magnitude of the slope. The profile in Fig. 2 is determined largely by the CCL design and performance, as the list above suggests. The pores of the CCL are (on 'average') much smaller than those of the GDL and the hydrophilic regions in the CCL cause water retention. Moreover, reaction is limited by the availability of catalyst, and can only occur at points of contact between the Pt and the electrolyte. The importance of the CCL explains the growing emphasis placed on its behaviour and design. Laboratory investigation of PEMFC is generally costly and difficult; for many problems of interest, mathematical (and computational) modelling has been a cost-effective aid (see Weber \& Newman, 2004, for a review).

Experimental studies have suggested that, during the preparation process, the carbon grains form clumps (called agglomerates) that are coated by the electrolyte (Weber \& Newman, 2004; Uchida et al., 1995, 1998; Siegel et al., 2003; Liu et al., 2003; Lister \& McLean, 2004; Mizuhata et al., 2004). It is also evident that this catalyst layer structure can be far from uniform. The theoretical problem is further compounded by a lack of certainty in the aspects of the physical behaviour, such as water formation, phase change, $\mathrm{p}^{+}$migration and the electrochemical kinetics. It is therefore inevitable that simplifications be made. Two of the earliest models can be found in Giner \& Hunter (1969) and Cutlip (1975), in which the CCL is assumed to be composed of single 'pores' that span the thickness of the layer. While such models lead to analytical solutions, largely through oversimplification of the equations, they neglect many of the important physical phenomena.

Models that employ the agglomerate description are found in, e.g. Siegel et al. (2003, 2004), Lin et al. (2004), Guo \& White (2004), Yin (2005) and Mazumder \& Cole (2004), in which the agglomeratelevel activity is incorporated into a homogeneous model by assuming that a film of electrolyte and/or liquid water surrounds each (spherical) agglomerate particle-motivated by the well-established theory of porous catalysts (see Chapter 3 of Aris, 1975). The three main variants are as follows: 
- Include external resistance to the $\mathrm{O}_{2}$ movement arising from the film(s); this yields an expression for the limiting current density as a function of the agglomerate characteristics (Siegel et al., 2003, 2004; Lin et al., 2004; Mazumder \& Cole, 2004).

- Include internal resistance to the $\mathrm{O}_{2}$ movement due to flooding of the agglomerates with electrolyte or liquid water (Guo \& White, 2004; Jaouen et al., 2002).

- Include both internal and external resistances, as described above (Yin, 2005; Sun et al., 2005).

Incorporation of mass-transport limitations a priori distinguishes these models from those of Song et al. (2004), Eikerling \& Kornyshev (1998), Eikerling \& Kornyshev (1999), Wang et al. (2004), Um et al. (2000), Genevey (2004) and Hsuen (2003), which treat the CCL as a homogeneous porous medium, i.e. each phase exists in each reference control volume and is specified solely by a volume fraction. Such models are not able to capture a limiting current density at all.

In the vast majority of cases, the main tool for theoretical investigation is numerical simulation, often multidimensional (Siegel et al., 2003, 2004; Sun et al., 2005; Mazumder \& Cole, 2004; Um et al., 2000) with the CCL model embedded in a much larger code. The influence of the CCL can be difficult to divorce from the influences of the other components represented in the model. The numerical methodology also requires that virtually all the parameter values are assumed fixed, with often order-ofmagnitude discrepancies in the values assumed. Investigation across (the extremely high dimensional) parameter space is necessarily a lengthy numerical undertaking. Such investigations are clearly more suited to approximate and asymptotic analysis.

Mathematical treatments, using analytical methods, are found in Giner \& Hunter (1969), Cutlip (1975), Eikerling \& Kornyshev (1998, 1999) and Hsuen (2003). Eikerling \& Kornyshev (1998, 1999), employ an isothermal homogeneous model, with a pseudo Maxwell-Stefan law for the $\mathrm{O}_{2}$ flux and Ohm's law for $\mathrm{p}^{+}$migration. They seek exact or implicit solutions in selected limits, which are justified through semi-heuristic arguments. Hsuen's (2003) approach is also homogeneous and isothermal, but includes an equation for the electrical potential and incorporates the GDL. However, his analysis rests on the (unjustified) assumption that either the $\mathrm{O}_{2}$ concentration or the electrolyte potential can be approximated by piecewise polynomial functions. There are significant gaps in this type of analysis, mainly in relation to liquid water movement (by capillary action), the multiphase nature of the structure, temperature variations and mass transfer between phases. In this paper, we tackle the last three in this list, focussing on relating the composition and microscopic details of the CCL structure to the profiles of $\mathrm{O}_{2}$ concentration, overpotential and temperature and ultimately to the performance measured through the current density. We pay particular attention to the transport of $\mathrm{O}_{2}$, as both a gaseous and dissolved species (in liquid water and electrolyte). It has a major impact on the performance of the cell at both low and high levels of humidity. Heat transport is of vital importance to the management of water and longevity of the cell. Even minor temperature increases (of a few degrees Celsius) can result in severe dehydration of the electrolyte, which makes it necessary to obtain a measure of control over its maximum value. Through the saturation pressure, temperature variations also drive deviations from equilibrium between the vapour and the liquid (unit relative humidity), and therefore drive condensation/evaporation.

In the Section 2, we present the model and the underlying assumptions. In Section 3, we derive a priori bounds and prove the existence of at least one solution. In Sections 4 and 5, important dimensionless parameters are identified, asymptotic solutions are constructed and numerical comparisons are provided. A summary follows in Section 6 where we also discuss the implications of the results and outline future work. 


\section{Mathematical model}

The model we derive is 1D in the 'through-plane' direction, i.e in a direction perpendicular to the $y z$ plane in Fig. 1. This is a justified assumption when the geometric area of the CCL is small and the flow rate (of delivery of $\mathrm{O}_{2}$ ) is high, as are usually the case for test cells. We also assume steady-state conditions, which are valid for the many so-called 'stationary applications' of PEMFC. Other important assumptions and details are now listed:

- Physical characterization of the CCL: The CCL structure that we assume is motivated by the large body of experimental results described in Section 1 (Weber \& Newman, 2004; Uchida et al., 1995, 1998; Siegel et al., 2003; Liu et al., 2003; Lister \& McLean, 2004-we refer in particular to the electron microscopy images in Figs 4-6 of Middelman (2002)). Three distinct length scales can be identified: (a) the length of the catalyst layer, from the membrane interface to the GDL $(10-100 \mu \mathrm{m})$; (b) the large-scale primary pores separating the agglomerates $(50-1000 \mathrm{~nm}$ ) and (c) the separation between the individual carbon grains or secondary pores $(1-50 \mathrm{~nm})$. We treat the CCL as a porous medium with a solid phase made up of Pt-supporting carbon particles and electrolyte. As in, e.g. Siegel et al. (2004), Guo \& White (2004), Yin (2005) and Sun et al. (2005), the carbon grains are assumed to form spherical clusters, which we call agglomerates (see Fig. 3), and between the agglomerates are the primary gas pores (constituting phase 1). The quantity of electrolyte inside the agglomerates depends on the preparation process and the precise volume is difficult to ascertain (Uchida et al., 1995; Lister \& McLean, 2004; Mizuhata et al., 2004). To take account of the

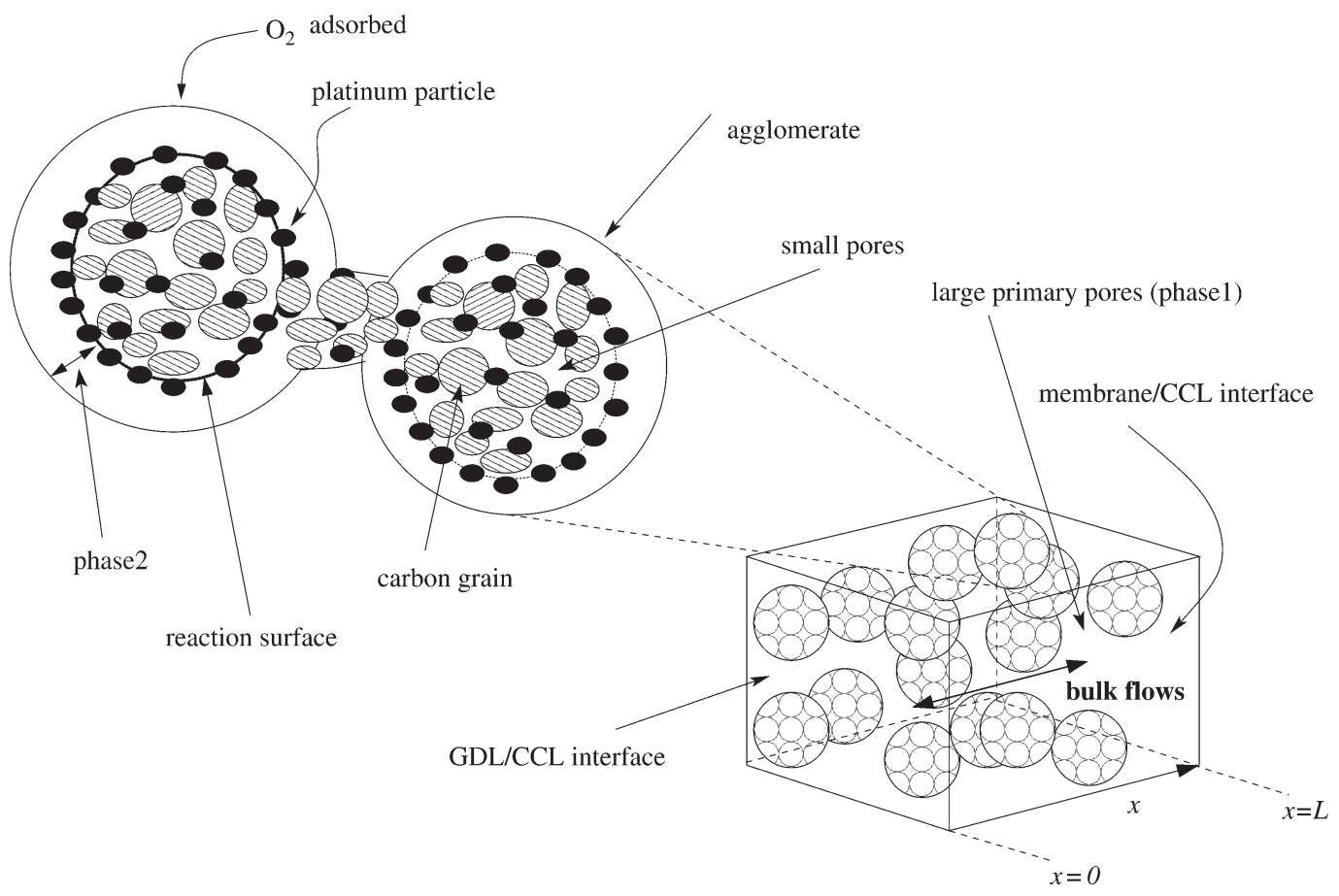

FIG. 3. The spherical agglomerate structure assumed. Oxygen diffuses through the phase 2 layer and reaction occurs at the surfaces of the agglomerates. 
electrolyte and liquid water that exists within the agglomerates, we assume that they are surrounded by homogeneously mixed regions of electrolyte, water and secondary gas pores (constituting phase 2 ); since the electrolyte is hydrophillic, it is reasonable to assume that any liquid water will coat its surface. Reaction is assumed to occur only on the inner surface of phase 2 . To ensure the free movement of $\mathrm{e}^{-}$and $\mathrm{p}^{+}$across the CCL, we assume a continuous matrix of carbon grains and sufficient contact between the carbon agglomerates. These connecting paths are assumed not to contribute to the reaction rate.

- Oxygen transport: Diffusion in phase 1 is assumed to be continuum. The $\mathrm{O}_{2}$ concentration is assumed to be exchanged from phase 1 to phase 2 by dissolution to water or electrolyte, with equilibrium described by Henry's law. The $\mathrm{O}_{2}$ in phase 2 then diffuses to the inner surface (see Fig. 3 ) where it reacts.

- Electro-neutrality: We assume electro-neutrality (the number of negative charges equals the number of positive charges) and a homogeneous distribution of charged sites in the electrolyte.

- Liquid water content: We assume equilibrium between liquid water and vapour with a constant volume of liquid water. These assumptions are perfectly justified at low values of relative humidity (the trend for PEMFC design), but at high levels will generally break down. Note also that the protonic conductivity is a function of water content; at a fixed electrolyte water content, it is approximately constant. These assumptions are further discussed in Section 6.

Other assumptions are that (a) there is a negligible ohmic potential drop in the electronically conducting carbon phase, justified by its high conductivity and (b) convective flow is negligible in the gas phase (see Mathias et al., 2003).

Let the concentrations of $\mathrm{O}_{2}$ in phases 1 and 2 be $C_{1}$ and $C_{2}$, respectively, i.e. $\mathrm{mol} /\left(\mathrm{m}^{3}\right.$ of phase 1 or 2). A mass balance in each phase yields

$$
\begin{gathered}
\epsilon_{1} D_{1} \frac{\mathrm{d}^{2} C_{1}}{\mathrm{~d} x^{2}}=\underbrace{h_{12}\left(H C_{1}-C_{2}\right)}_{\text {interfacial mass transfer }}, \\
\epsilon_{2} D_{2} \frac{\mathrm{d}^{2} C_{2}}{\mathrm{~d} x^{2}}=\underbrace{\frac{1}{4} \mathcal{R}\left(\eta_{\mathrm{c}}, T, C_{2}^{S}\right)}_{\text {reactant consumption }}-h_{12}\left(H C_{1}-C_{2}\right),
\end{gathered}
$$

where $x$ is the space variable (see Fig. 1), $\epsilon_{1}\left(\epsilon_{2}\right)$ is the volume fraction of phase 1 (phase 2), $D_{1}$ $\left(D_{2}\right)$ is the effective molecular diffusion coefficient for $\mathrm{O}_{2}$ through phase 1 (phase 2), $\mathcal{R}\left(\eta_{\mathrm{c}}, T, C_{2}^{S}\right)$ is the $\left(\mathrm{O}_{2}\right.$ reduction) reaction rate, $h_{12}$ is the interfacial mass-transfer coefficient from phase 1 to 2 and $H$ is Henry's constant. The bulk diffusive flux of $\mathrm{O}_{2}$ in phase 1 is balanced by the amount adsorbed into phase 2. The effective diffusion coefficient for $\mathrm{O}_{2}$ in phase $2, D_{2}$, is an unknown function of the coefficients in the constitutive components. The implications of this are quite important; the coefficient for the pores differs by several orders of magnitude from the other two. $D_{2}$ will generally depend on the geometry and composition of phase 2 , the size of the secondary pores and the diffusivities in the gas and solid phases (respectively, the upper and lower bounds in Table 1). Though $D_{2}$ is likely to fall somewhere between these values, we will later exploit them in an asymptotic analysis as the limits of weak and strong diffusion limitation. 
TABLE 1 A list of parameters and their values, where available. Values are taken from Siegel et al.(2003), Um et al.(2000), Mazumder \& Cole (2004), Lin et al. (2004), Song et al. (2004), Yin (2005), Sun et al. (2005), Huang \& White (2000), Middelman (2002), Berg et al. (2004) and Ziegler et al. (2004)

\begin{tabular}{|c|c|c|}
\hline Symbol & Quantity & Size \\
\hline$L$ & Catalyst layer width & $10^{-5}$ to $10^{-4} \mathrm{~m}$ \\
\hline$D_{1}$ & Effective diffusion coefficient of phase 1 & $10 \times 10^{-4}$ to $10^{-5} \mathrm{~m}^{2} \mathrm{~s}^{-1}$ \\
\hline$D_{2}$ & Effective diffusion coefficient of $\mathrm{O}_{2}$ in phase 2 & $10^{-6}$ to $10^{-10} \mathrm{~m}^{2} \mathrm{~s}^{-1}$ \\
\hline${ }^{\dagger} \sigma_{\mathrm{e}}$ & Effective protonic conductivity & $1-10 \mathrm{~S} \mathrm{~m}^{-1}$ \\
\hline$c_{\mathrm{W}}$ & Free water concentration of phase 2 & $1-40 \mathrm{~mol} \mathrm{~m}^{-3}$ \\
\hline$\epsilon_{1}$ & Volume fraction of phase 1 & $0.3-0.6$ \\
\hline$\epsilon_{2}$ & Volume fraction of phase 2 & $0.1-0.5$ \\
\hline$\epsilon_{\mathrm{W}}$ & Volume fraction of water in phase 2 & $0.1-0.4$ \\
\hline$-\delta S$ & Entropy associated with ORR & $236 \mathrm{~J} \mathrm{~mol}^{-1} \mathrm{~K}^{-1}$ \\
\hline$c_{O, \text { ref }}$ & Reference $\mathrm{O}_{2}$ concentration & $0.6-41 \mathrm{~mol} \mathrm{~m}^{-3}$ \\
\hline$i_{O_{2}, \mathrm{c}}$ & Reference cathode exchange current density & $10^{2}$ to $10^{-4} \mathrm{~A} \mathrm{~m}^{-2}$ \\
\hline$\alpha_{\mathrm{c}}$ & Cathodic transfer coefficient & $0.5-2$ \\
\hline$R_{\mathrm{ag}}$ & Agglomerate radius & $0.5-3 \mu \mathrm{m}$ \\
\hline$\delta$ & Electrolyte film thickness & $\mathrm{m}$ \\
\hline$N$ & Number of agglomerates per unit volume & $10^{18}$ to $10^{15} \mathrm{~m}^{-3}$ \\
\hline $\bar{T}$ & GDL temperature & $370-390 \mathrm{~K}$ \\
\hline$E_{0}$ & Open circuit potential & $1 \mathrm{~V}$ \\
\hline$U_{\mathrm{c}}$ & Carbon phase potential & $0-1 \mathrm{~V}$ \\
\hline$\beta$ & Platinum surface per volume & $10^{6}$ to $10^{7} \mathrm{~m}^{-1}$ \\
\hline $\bar{C}$ & $\mathrm{O}_{2}$ concentration at GDL interface & $\mathrm{mol} \mathrm{m}^{-3}$ \\
\hline $\bar{\phi}$ & Membrane potential at membrane interface & $\mathrm{V}$ \\
\hline$h_{12}$ & $\mathrm{O}_{2}$ mass transfer rate & $10^{2}$ to $10^{4} \mathrm{~s}^{-1}$ \\
\hline$H$ & Dimensionless Henry's law constant & $0.032-2$ \\
\hline $\bar{\lambda}$ & Effective thermal conductivity of CCL & $0.2-2 \mathrm{~W} \mathrm{~m}^{-1} \mathrm{~K}^{-1}$ \\
\hline
\end{tabular}

${ }^{\dagger}$ Based on a membrane water content of below seven and operating temperatures in the range 303-363 K.

With the assumption of electro-neutrality, Ohm's law yields the following equation for the potential in phase $2, \phi$ :

$$
\frac{\mathrm{d}}{\mathrm{d} x} \underbrace{\left(\frac{\epsilon_{\mathrm{w}} \sigma_{\mathrm{e}}}{F} \frac{\mathrm{d} \phi}{\mathrm{d} x}\right)}_{\mathrm{p}^{+} \text {migration }}=\underbrace{\mathcal{R}\left(\eta_{\mathrm{c}}, T, C_{2}^{S}\right)}_{\mathrm{p}^{+} \text {consumption }},
$$

where $\epsilon_{\mathrm{W}}$ is the combined volume fraction of water and electrolyte in phase $2, \sigma_{\mathrm{e}}$ is the effective protonic conductivity of phase 2 and $F$ is Faraday's constant $\left(9.643 \times 10^{4} \mathrm{~A} \mathrm{~s} \mathrm{~mol}^{-1}\right)$.

An equation for the temperature, $T$, is derived from an energy balance

$$
\bar{\lambda} \frac{\mathrm{d}^{2} T}{\mathrm{~d} x^{2}}=-\underbrace{\left(\frac{(-\delta s) T}{n_{\mathrm{e}}}+F \eta_{\mathrm{c}}\right) \mathcal{R}\left(\eta_{\mathrm{c}}, T, C_{2}^{S}\right)}_{\text {heat generation }} .
$$


The right-hand side is a combination of the heat of electrochemical reaction (first term), with entropy $-(\delta s)$, and heat arising from activation losses. $n_{\mathrm{e}}=4$ is the number of $\mathrm{e}^{-}$transferred in the reaction and $\bar{\lambda}$ is an effective thermal conductivity. We have neglected ohmic heating which is much weaker than heating from reaction and activation losses.

The ORR rate, in $\mathrm{mol} /\left(\mathrm{m}^{3}\right.$ of bulk $\left.\cdot \mathrm{s}\right)$, is given by the Tafel law, which assumes first-order kinetics in $\mathrm{O}_{2}$ concentration and that the 'backward' reaction is negligible:

$$
\mathcal{R}\left(\eta_{\mathrm{c}}, T, C_{2}^{s}\right)=\frac{\beta i_{O_{2}, \mathrm{c}}}{F c_{O_{2}, \mathrm{ref}}} \epsilon_{2} C_{2}^{s} \exp \left(\frac{\alpha_{\mathrm{c}} F \eta_{\mathrm{c}}}{R T}\right),
$$

where $i_{O_{2}, \mathrm{c}}$ is the exchange current density, $c_{O_{2}}$, ref is a reference $\mathrm{O}_{2}$ concentration, $\alpha_{\mathrm{c}}$ is a transfer coefficient, $\beta$ is the surface area of Pt per unit volume of bulk, $\eta_{\mathrm{c}}$ is the overpotential and $R$ is the universal gas constant $\left(8.314 \mathrm{~J} \mathrm{~mol}^{-1} \mathrm{~K}^{-1}\right) . \beta$ is a function of the $\mathrm{Pt}$ loading (Pt surface area per unit mass of Pt) and the CCL thickness. The overpotential, $\eta_{\mathrm{c}}$, is defined through the relationship

$$
\eta_{\mathrm{c}}=E_{0}-U_{\mathrm{c}}-\phi
$$

where $U_{\mathrm{c}}$ is the potential of the carbon matrix, assumed constant, and $E_{0}$ is the reference open-circuit potential of the cathode.

\subsection{Expression for $C_{2}^{S}$ and the limiting current density}

The $\mathrm{O}_{2}$ concentration in (2.5), $C_{2}^{s}$, is the value at the surfaces of the carbon agglomerates, i.e. at the reaction location. The value of $C_{2}^{s}$ can be related to the bulk concentration in phase $2, C_{2}$, by balancing the rate of reaction with the diffusion of $\mathrm{O}_{2}$ to the surfaces of the agglomerates (at steady state). This mass balance can be approximately expressed as follows:

$$
\gamma^{\prime}\left(C_{2}-C_{2}^{s}\right)=\frac{1}{4} \mathcal{R}\left(\eta_{\mathrm{c}}, T, C_{2}^{s}\right)
$$

where $\gamma^{\prime}$ is a measure of the rate of $\mathrm{O}_{2}$ diffusion through phase 2 to the surface of the agglomerates (in s$\left.{ }^{-1}\right)$. Using the definition of $\mathcal{R}\left(\eta_{\mathrm{c}}, T, C_{2}^{S}\right)$ and solving the resulting equation for $C_{2}^{s}$ then yields

$$
C_{2}^{S}=\gamma^{\prime} C_{2}\left(\gamma^{\prime}+\frac{\epsilon_{2} \beta i_{O_{2}, \mathrm{c}}}{4 F c_{O_{2}, \text { ref }}} \mathrm{e}^{\alpha_{\mathrm{c}} F \eta_{\mathrm{c}} / R T}\right)^{-1},
$$

so that the final form of the reaction rate is

$$
\mathcal{R}\left(\eta_{\mathrm{c}}, T, C_{2}^{S}\right) \equiv \mathcal{R}\left(\eta_{\mathrm{c}}, T, C_{2}\right)=\frac{\epsilon_{2} \beta i_{O_{2}, \mathrm{c}}}{F c_{O_{2}, \mathrm{ref}}} \gamma^{\prime} C_{2} \mathrm{e}^{\alpha_{\mathrm{c}} F \eta_{\mathrm{c}} / R T}\left(\gamma^{\prime}+\frac{\epsilon_{2} \beta i_{O_{2}, \mathrm{c}}}{4 F c_{O_{2}, \text { ref }}} \mathrm{e}^{\alpha_{\mathrm{c}} F \eta_{\mathrm{c}} / R T}\right)^{-1} .
$$

In order to relate $\gamma^{\prime}$ to the microscopic properties of the CCL, we define a phase 2 thickness, $\delta$, an agglomerate radius, $R_{\mathrm{ag}}$, and the number of agglomerates per unit volume, $N$, to obtain

$$
\gamma^{\prime}\left(C_{2}-C_{2}^{S}\right) \approx 4 \pi R_{\mathrm{ag}}^{2} N \times \frac{D_{2}}{\delta}\left(C_{2}-C_{2}^{S}\right),
$$

where the first term on the right-hand side is the specific surface area of the agglomerates and the second term is the molar flux of $\mathrm{O}_{2}$ to the agglomerate surfaces. We therefore have the approximate expression

$$
\gamma^{\prime}=\frac{4 \pi R_{\mathrm{ag}}^{2} N D_{2}}{\delta} \text {. }
$$


Note that the quantity $N$ is a function of not just the agglomerate radius but also the distribution (the more densely packed the agglomerates, the larger $N)$. The phase 2 thickness, $\delta$, can approach zero independently of $\epsilon_{2}$ by increasing $N$ according to $\epsilon_{2}=4 \pi N\left(\left(R_{\mathrm{ag}}+\delta\right)^{3}-R_{\mathrm{ag}}^{3}\right) / 3$. As $\epsilon_{2} \rightarrow 0$, the reaction rate approaches zero, i.e. reaction can only occur in the presence of $\mathrm{p}^{+}$.

The current flow is the reverse of the $\mathrm{p}^{+}$flow; the current density, $I^{\prime} \mathrm{A} \mathrm{m}^{-2}$, is therefore

$$
I^{\prime}=4 F r \gamma^{\prime} \int_{0}^{L} \frac{C_{2} \mathrm{e}^{\alpha_{\mathrm{c}} F \eta_{\mathrm{c}} / R T}}{\gamma^{\prime}+r \mathrm{e}^{\alpha_{\mathrm{c}} F \eta_{\mathrm{c}} / R T}} \mathrm{~d} x, \quad \text { where } r=\frac{\epsilon_{2} \beta i_{O_{2}, c}}{4 F c_{O_{2}, \text { ref }}} .
$$

As $\eta_{\mathrm{c}} \rightarrow \infty$, the cell voltage approaches $-\infty$. In this limit, at fixed values of the other parameters, we find that

$$
I^{\prime} \rightarrow I_{\lim }^{\prime} \equiv 4 F \gamma^{\prime} \int_{0}^{L} C_{2} \mathrm{~d} x \leqslant 4 F \gamma^{\prime} \bar{C} L
$$

where $I_{\text {lim }}^{\prime}$ is the limiting value of the current density (the mass-transport limit in Fig. 2). So we see immediately that the current density is limited by the concentration of $\mathrm{O}_{2}$ entering the system, the width of the CCL and the size of $\gamma^{\prime}$. The latter, defined in (2.11), is proportional to the specific surface area of the agglomerates, as is the quantity $\beta$, and the specific surface area of Pt. So, both $I^{\prime}$ and $I_{\text {lim }}^{\prime}$ increase with increasing $\beta$, as one would expect.

\subsection{Boundary conditions}

At the membrane/CCL interface, placed at $x=L$ (see Fig. 1), we prescribe the overpotential, $\bar{\eta}_{\mathrm{c}}$, with the assumption that the membrane/electrolyte potential is zero at that point. Typically, the latter is close to zero, and since our focus is on the CCL, there is no loss of generality in assuming that it is identically zero (this is common approximation). The 'cell voltage' can then be approximated by the carbon-phase potential, $U_{\mathrm{c}}$, which is given by $U_{\mathrm{c}}=E_{0}-\bar{\eta}_{\mathrm{c}}$. Since $\mathrm{O}_{2}$ cannot penetrate the membrane in the gas phase, the $\mathrm{O}_{2}$ concentrations satisfy a zero-flux (Neumann) condition. For simplicity, we make the same assumption for phase 2; in reality the flux is small. The heat flux across the boundary is also assumed to be zero:

$$
\eta_{\mathrm{c}}=\bar{\eta}_{\mathrm{c}}, \quad \frac{\mathrm{d} C_{2}}{\mathrm{~d} x}=\frac{\mathrm{d} C_{1}}{\mathrm{~d} x}=0, \quad \frac{\mathrm{d} T}{\mathrm{~d} x}=0
$$

At the GDL/CCL interface, placed at $x=0$, the $\mathrm{O}_{2}$ in phase 1 enters the CCL at a known concentration, $\bar{C}$, which can be approximated from the value at the channel in a straightforward manner. It is therefore a function of the GDL properties (in addition to the channel value). Also at $x=0$, we assume that the $\mathrm{O}_{2}$ in the electrolyte and water is in equilibrium with that in phase 2 (Henry's law). $\mathrm{p}^{+}$cannot penetrate the GDL and are therefore subject to a zero-flux condition at $x=0$. As with the $\mathrm{O}_{2}$ concentration in phase 1 , the temperature at $x=0$ is approximated from the value in the channel:

$$
C_{2}=H C_{1}=\bar{C}, \quad \frac{\mathrm{d} \phi}{\mathrm{d} x}=0, \quad T(0)=\bar{T}
$$

A list of parameters and their values can be found in Table 1. We further refer to the comments made at the beginning of Section 4 . 


\subsection{Nondimensionalization}

It is convenient for the analysis that follows to nondimensionalize the boundary-value problem (BVP), according to

$$
\begin{gathered}
x=x_{\mathrm{c}} z, \quad \eta_{\mathrm{c}}=\phi_{\mathrm{c}} \psi, \quad T=\bar{T} \tau, \quad C_{2}=\bar{C} X_{2}, \quad C_{1}=\bar{C} X_{1}, \quad I^{\prime}=I \mathcal{I}, \quad l=L / x_{\mathrm{c}}, \\
x_{\mathrm{c}}^{2}=\frac{\epsilon_{2} D_{2}}{\gamma^{\prime}}, \quad \gamma=\frac{4 \gamma^{\prime} F c_{O_{2}, \mathrm{ref}}}{\epsilon_{2} \beta i_{O_{2}, \mathrm{c}}}, \quad \kappa=\frac{4 F \epsilon_{2} D_{2} \bar{C}}{\epsilon_{\mathrm{w}} \sigma_{\mathrm{e}} \phi_{\mathrm{c}}}, \quad h=\frac{x_{\mathrm{c}}^{2} h_{12}}{\epsilon_{2} D_{2}}, \quad \eta=\frac{\epsilon_{2} D_{2}}{\epsilon_{1} D_{1}}, \\
s=\frac{-(\delta S) \bar{T}}{4 F \phi_{\mathrm{c}}}, \quad \mathcal{I}=\frac{x_{\mathrm{c}} \bar{C} \epsilon_{2} \beta i_{O_{2}, \mathrm{c}}}{c_{O_{2}, \mathrm{ref}}}, \quad \Delta=\frac{4 \epsilon_{2} D_{2} \bar{C} R}{\bar{\lambda} \alpha_{\mathrm{c}}}, \quad \bar{\psi}=\frac{\bar{\eta}_{\mathrm{c}}}{\phi_{\mathrm{c}}}, \quad \phi_{\mathrm{c}}=\frac{R \bar{T}}{\alpha_{\mathrm{c}} F},
\end{gathered}
$$

to yield

$$
\begin{gathered}
X_{2}^{\prime \prime}=\frac{\gamma X_{2} \mathrm{e}^{\psi / \tau}}{\gamma+\mathrm{e}^{\psi / \tau}}-h\left(H X_{1}-X_{2}\right), \\
X_{1}^{\prime \prime}=\frac{h}{\eta}\left(H X_{1}-X_{2}\right), \\
\psi^{\prime \prime}=-\frac{\kappa \gamma X_{2} \mathrm{e}^{\psi / \tau}}{\gamma+\mathrm{e}^{\psi / \tau}}, \\
\tau^{\prime \prime}=-(s \tau+\psi) \frac{\Delta \gamma X_{2} \mathrm{e}^{\psi / \tau}}{\gamma+\mathrm{e}^{\psi / \tau}}, \\
\int_{0}^{l} \frac{X_{2} \mathrm{e}^{\psi / \tau}}{\gamma+\mathrm{e}^{\psi / \tau}} \mathrm{d} z \quad \text { with } I_{\lim } \equiv \gamma \int_{0}^{l} X_{2} \mathrm{~d} z, \\
\psi(l)=\bar{\psi}, \quad \tau(0)=1, \quad X_{2}^{\prime}(l)=X_{1}^{\prime}(l)=0, \\
X_{2}(0)=H X_{1}(0)=1, \quad \psi^{\prime}(0)=\tau^{\prime}(l)=0,
\end{gathered}
$$

where primes denote differentiation w.r.t. $z$. Note that the dimensionless potential $\psi$ is actually a dimensionless overpotential. The reference length scale, $x_{\mathrm{c}}$, is a function of the ratio of bulk molecular diffusion of $\mathrm{O}_{2}$ through phase 2 and the diffusion rate to the surfaces of the agglomerates. The latter quantity, $\gamma$, appears to be inversely proportional to the Pt surface area per unit volume, $\beta$. However, since $\beta$ is proportional to the specific surface area of the agglomerates, there is in effect no dependence on $\beta$. The current density, including the limiting value, does, however, depend on $\beta$ through the reference value $\mathcal{I}$. We can further infer that the reaction rate, and therefore the solution, is heavily dependent on the size of $\gamma$. As $\gamma \rightarrow \infty$, the classical Tafel reaction rate is recovered. On the other hand, for $\gamma \rightarrow 0$, the reaction depends entirely on the $\mathrm{O}_{2}$ concentration.

\section{A priori bounds and existence}

For the isothermal equivalent to (2.17), a priori bounds are straightforward to derive from maximum principles, e.g.

$$
0 \leqslant X_{2} \leqslant H, \quad \bar{\psi} \leqslant \psi \leqslant \bar{\psi}-\frac{H \kappa \gamma}{2}\left(z^{2}-l^{2}\right)
$$


in the limit $h \rightarrow \infty$. Note that there is no restriction on the width of the catalyst layer, $l$ (in fact these bounds are uniformly continuous in $l$ ). For the nonisothermal model (2.17), we shall discover that analysis of the temperature problem places a restriction on $l$ (in deriving a maximum principle). Furthermore, we are able to derive bounds on the solution, particularly the maximum values that can be assumed at the endpoints $z=0$ and $z=L$. Using the Leray-Schauder fixed-point theorem, we can then demonstrate that (2.17) admits a solution.

LEMMA 3.1 (A priori bounds) Assume that the condition $l^{2}<2 /(H s \Delta \gamma)$ holds. Then, classical solutions to (2.17) satisfy the following estimates:

$$
\begin{gathered}
0 \leqslant X_{1} \leqslant 1, \quad \frac{H}{\cosh (l \sqrt{\gamma+h})} \leqslant X_{2} \leqslant H, \quad H X_{1} \geqslant X_{2}, \\
1 \leqslant \tau \leqslant \tau_{\max } \equiv 1+\frac{H \Delta \gamma l^{2}(s+\bar{\psi})}{2-l^{2} s H \Delta \gamma}, \\
\bar{\psi} \leqslant \psi \leqslant \bar{\psi}-\frac{H \kappa \gamma}{2}\left(z^{2}-l^{2}\right) \leqslant \bar{\psi}+\frac{H \kappa \gamma l^{2}}{2} \equiv \psi_{\max } .
\end{gathered}
$$

Proof. We first demonstrate that $X_{2}$ and $X_{1}$ are nonnegative. Note that the function $\mathrm{e}^{\psi / \tau} /\left(\gamma+\mathrm{e}^{\psi / \tau}\right)$ is bounded above by unity, for all values of $\psi$ and $\tau$ in $\mathbb{R}$. Let us assume that $X_{2}=0$ at an interior point, $z=z_{*}, X_{1}$ and $X_{2}$ are positive in $\left[0, z_{*}\right)$ and $X_{2}^{\prime}\left(z_{*}\right)<0$. From (2.17a) and (2.17b), we find that in $\left[0, z_{*}\right)$,

$$
\left(H X_{1}-X_{2}\right)^{\prime \prime}-\frac{h}{\eta}(H+\eta)\left(H X_{1}-X_{2}\right)=-\frac{\gamma X_{2} \mathrm{e}^{\psi / \tau}}{\gamma+\mathrm{e}^{\psi / \tau}}<0
$$

so that $H X_{1}-X_{2}$ cannot assume a nonpositive interior minimum. Now, since $\left(H X_{1}-X_{2}\right)(0)=0$ and $\left(H X_{1}-X_{2}\right)\left(z_{*}\right)>0, H X_{1}-X_{2} \geqslant 0$ in $\left[0, z_{*}\right]$. By continuity, there exists a region $\Omega_{1} \subset\left[z_{*}, l\right]$ in which $X_{1} \geqslant 0$ and $X_{2} \leqslant 0$ and therefore

$$
X_{2}^{\prime \prime}-\left(\frac{\gamma \mathrm{e}^{\psi / \tau}}{\gamma+\mathrm{e}^{\psi / \tau}}+h\right) X_{2}=-h H X_{1} \leqslant 0, \quad X_{1}^{\prime \prime}-\frac{h H}{\eta} X_{1}=-\frac{h}{\eta} X_{2} \geqslant 0, \quad x \in \Omega_{1} .
$$

Suppose that $X_{1}$ is monotonically increasing in $\left[0, z_{*}\right]$. From (3.3), $X_{2}$ cannot attain a nonpositive interior minimum and $X_{1}$ cannot attain an interior nonnegative maximum in $\Omega_{1}$. Therefore, $\Omega_{1}=\left[z_{*}, l\right]$ and $X_{2}^{\prime \prime} \leqslant 0$ in $\Omega_{1}$. Integrating this inequality over $\Omega_{1}$, we find that $X_{2}^{\prime}\left(z_{*}\right)>0$, which is a contradiction. Thus, $X_{1}$ is monotonically nonincreasing in $\left[0, z_{*}\right]$. If $H X_{1}-X_{2}$ remains positive, from (2.17a), we find that $X_{2}$ cannot attain a nonpositive minimum in the region $\left[z_{*}, l\right]$. Integrating $X_{2}^{\prime \prime} \leqslant 0$ over $\left[z_{*}, l\right]$ once again leads to a contradiction. There must therefore exist a point $z^{*}$ at which $H X_{1}-X_{2}=0$, with $H X_{1}-X_{2} \geqslant 0$ in $\left[0, z^{*}\right]$ and, by continuity, $H X_{1}-X_{2} \leqslant 0$ for some region $\Omega_{2} \subset\left[z^{*}, l\right]$. If $H X_{1}-X_{2}$ remains nonpositive in $\Omega_{2}, X_{1}^{\prime \prime} \leqslant 0$ in the latter region. Integrating this expression over $\Omega_{2}$ yields $X_{1}^{\prime}\left(z^{*}\right)>0$, a contradiction. Therefore, there must exist a point $z_{*}^{*} \in \Omega_{2}$ at which $H X_{1}-X_{2}=0$ and $\left(H X_{1}-X_{2}\right)^{\prime}>0$. In the region $\Omega_{2}=\left[z_{*}, z^{*}\right]$,

$$
X_{2}^{\prime \prime}-\frac{\gamma \mathrm{e}^{\psi / \tau}}{\gamma+\mathrm{e}^{\psi / \tau}} X_{2} \geqslant 0, \quad X_{1}^{\prime \prime} \leqslant 0,
$$

so $X_{2}$ cannot attain a nonnegative maximum and $X_{1}$ cannot attain a minimum in the interior of $\left[z_{*}, z^{*}\right]$. Both $X_{1}$ and $X_{2}$ are therefore negative in the latter region and in order that $\left(H X_{1}-X_{2}\right)\left(z^{*}\right)=0$, 
$X_{2}$ must attain a negative maximum in $\left[z_{*}, z^{*}\right]$. By continuity, there is a region $\Omega_{3} \subset\left[z_{*}^{*}, l\right]$ in which $H X_{1}-X_{2} \geqslant 0$ with both $X_{1}$ and $X_{2}$ negative. In this region, $X_{1}^{\prime \prime} \geqslant 0$ and $X_{2}^{\prime \prime} \leqslant 0$, so that $X_{1}$ cannot attain a maximum and $X_{2}$ a minimum, and $\left(H X_{1}-X_{2}\right)(z)-\left(H X_{1}-X_{2}\right)\left(z^{*}\right)>0, \forall z \in \Omega_{3}$. This demands that $\Omega_{3}=\left[z_{*}^{*}, l\right]$. From (3.2), we now obtain $\left(H X_{1}-X_{2}\right)^{\prime \prime} \geqslant 0, x \in \Omega_{3}$, suggesting that $\left(H X_{1}-X_{2}\right)^{\prime}\left(z_{*}^{*}\right)<0$, which is a contradiction. Therefore, $X_{2}$ is positive in $[0, l]$.

Let us now assume that $X_{2}$ is zero at an interior point $z_{*}$, with $X_{1} \geqslant 0$ and $X_{2}>0$ in $\left[0, z_{*}\right]$. Examining the equations, there are two possibilities (i) $H X_{1}-X_{2}<0$ in $\left[0, z_{*}\right]$ and (ii) $H X_{1}-X_{2}$ has a positive maximum in $\left[0, z_{*}\right]$. Let us assume case (i) holds. In the region $\left[0, z_{*}\right], X_{1}^{\prime \prime}<0, X_{2}^{\prime \prime}>0$ and $\left(H X_{1}-X_{2}\right)^{\prime \prime}<0$, so that there is no minimum in $X_{1}$, maximum in $X_{2}$ or minimum in $H X_{1}-X_{2}$ in $\left(0, z_{*}\right)$. Both $X_{1}$ and $X_{2}$ must therefore decrease monotonically in the latter region. If $X_{2}$ remains positive, $\eta\left(H X_{1}-X_{2}\right)^{\prime \prime}-h(H+\eta)\left(H X_{1}-X_{2}\right)<0$ in $[0, l]$, so that $H X_{1}-X_{2}$ cannot attain a nonpositive minimum, and is therefore negative in $[0, l]$. Integrating the resulting identity, $\left(H X_{1}-\right.$ $\left.X_{2}\right)^{\prime \prime}<0$, over $[0, l]$ yields $\left(H X_{1}-X_{2}\right)^{\prime}(0)>0$, which is a contradiction. Therefore, there exists a point, $z_{1}$, at which $X_{2}=0$, but this cannot happen unless $X_{1}<0$ at some point $z^{*}<z_{1}$. In [ $z^{*}, z_{1}$ ], $X_{2}^{\prime}$ increases and $X_{1}^{\prime}$ decreases monotonically. By continuity, there is a region $\Omega_{1} \subset\left[z_{1}, l\right]$ in which $H X_{1}-X_{2}$ is negative. If $\Omega_{1}=\left[z_{1}, l\right], X_{1}^{\prime \prime} \leqslant 0$ in $[0, l]$, which leads to a contradiction, $X_{1}^{\prime}(0)>0$. This implies the existence of a point $z_{*}^{*}$ at which $H X_{1}-X_{2}=0$ and a region $\Omega_{2} \subset\left[z_{*}^{*}, l\right]$ in which $H X_{1}-X_{2} \geqslant 0$ and $X_{2}<0$. Arguing as above, we find that necessarily $\Omega_{2}=\left[z_{*}^{*}, l\right]$. Integrating the inequality $\left(H X_{1}-X_{2}\right)^{\prime \prime}>0$ over $\left[z_{*}^{*}, l\right]$, we find that $\left(H X_{1}-X_{2}\right)^{\prime}\left(z_{*}^{*}\right)<0$, a contradiction. A similar line of reasoning rules out possibility (ii). This proves that $X_{1}$ and $X_{2}$ are nonnegative.

From (2.17a) and (2.17b), and the nonnegativity of $X_{2}$, we now obtain

$$
\eta\left(H X_{1}-X_{2}\right)^{\prime \prime}-h(H+\eta)\left(H X_{1}-X_{2}\right) \leqslant 0, \quad x \in[0, l],
$$

so that $H X_{1}-X_{2}$ cannot attain a nonpositive minimum. Suppose that $H X_{1}-X_{2}=0$ at some $z_{*} \in(0, l)$. Since it cannot attain a negative minimum, $H X_{1}-X_{2} \leqslant 0$ in $\left[z_{*}, l\right]$. But then integrating $\left(H X_{1}-\right.$ $\left.X_{2}\right)^{\prime \prime}<0$ over $\left[z_{*}, l\right]$ yields $\left(H X_{1}-X_{2}\right)^{\prime}\left(z_{*}\right)>0$, a contradiction. Therefore, $X_{2} \leqslant H X_{1}$, so that $X_{1}^{\prime \prime} \geqslant 0$, whereupon $X_{1} \leqslant 1$ from Hopf's lemma, and therefore $X_{2} \leqslant H$. Since $X_{2} \geqslant 0$ and $X_{1} \geqslant 0$, the inequality $X_{2}^{\prime \prime}-(\gamma+h) X_{2} \leqslant 0$ holds. Therefore, the solution to the problem

$$
\underline{X}_{2}^{\prime \prime}-(\gamma+h) \underline{X}_{2}=0, \quad \underline{X}_{2}(0)-H=\underline{X}_{2}^{\prime}(l)=0
$$

is a lower solution to $(2.17 \mathrm{a}),(2.17 \mathrm{f})$, i.e. $X_{2} \geqslant \underline{X}_{2}$. This yields

$$
X_{2} \geqslant H \frac{\cosh ((z-l) \sqrt{\gamma+h})}{\cosh (l \sqrt{\gamma+h})}
$$

which completes the proof of the first part of (3.1).

We move now to overpotential and temperature. Using the upper and lower bounds for $X_{2}$ in (2.17a), we are led to $\psi \geqslant \bar{\psi}$ and

$$
\psi^{\prime \prime} \geqslant-\kappa \gamma H
$$

which leads to

$$
\bar{\psi} \leqslant \psi \leqslant \bar{\psi}+\frac{H \kappa \gamma}{2}\left(l^{2}-z^{2}\right)
$$

To bound $\tau$ from above, we first note that the classical generalized maximum principles do not apply because of the positivity of the term multiplying $\tau$ on the right-hand side of (2.17d). To overcome this 
difficulty, we will need the function $w(z)>0$, constrained to satisfy

$$
w^{\prime \prime}+\Delta \gamma s H w<0, \quad z \in(0, l) .
$$

A suitable function is $w(z)=1-k z^{2}$, where $k>\Delta \gamma s H / 2$, and we must assume that

$$
l<\frac{1}{\sqrt{k}}<\sqrt{\frac{2}{\Delta \gamma s H}} .
$$

Next, from the positivity of $\tau$,

$$
\tau^{\prime \prime}=-(s \tau+\psi) \frac{\Delta \gamma X_{2} \mathrm{e}^{\psi / \tau}}{\gamma+\mathrm{e}^{\psi / \tau}}>-\left(s \tau+\psi_{\max }\right) H \Delta \gamma, \quad z \in(0, l),
$$

where $\psi_{\max }$ is defined in (3.1). We can demonstrate that functions satisfying

$$
\tau_{2}^{\prime \prime}=-\left(s \tau_{2}+\psi_{\max }\right) H \Delta \gamma, \quad z \in(0, l), \quad \tau_{2}(0)=1, \quad \tau_{2}^{\prime}(l)=0
$$

are upper solutions to $(2.17 \mathrm{~d})$, i.e. they satisfy $\tau_{2} \geqslant \tau$ in $[0, l]$. Furthermore, we can demonstrate that functions satisfying

$$
\tau_{m}^{\prime \prime} \leqslant-\left(s \tau_{m}+\psi_{\max }\right) H \Delta \gamma, \quad z \in(0, l), \quad \tau_{2}(0) \geqslant 1, \quad \tau_{2}^{\prime}(l) \geqslant 0
$$

are upper solutions to the problem in $\tau_{2}$, and therefore upper solutions to $\tau$. The proof of these assertions requires the existence of the function $w(x)$ and hence the constraint on the size of the domain (the size of $l$ ). Essentially, it can be shown that $\tau / w, \tau_{2} / w$ and $\tau_{m} / w$ yield to a maximum principle. A suitable $\tau_{m}$ is found in the form

$$
\tau_{m}=1+a z(2 l-z), \quad \text { where } a \geqslant \frac{H \Delta \gamma\left(s+\psi_{\max }\right)}{2-l^{2} s H \Delta \gamma},
$$

and additionally, we require that $l^{2}<2 /(\Delta \gamma s H)$, which is the same as Condition (3.11). Alternatively, we can solve Problem (3.13) directly to obtain a sharper bound, but since it is much more difficult to interpret, given the upper bound on $l$, we will use (3.15) in the sequel. Finally, from the positivity of $\tau, \tau^{\prime \prime}<0$ so that $\tau \geqslant 1$ in $[0, l]$.

REMARK 3.1 (On the proof of Lemma 3.1) A restriction of the type (3.11) on the size of $l$, as a function of the other parameters, is necessary in order to establish a maximum principle. It may be possible to increase the size of $l$ through a different choice of the function $w(z)$. It is not possible, however, to split the domain and employ separate $w$ functions in each subdomain, as in the initial-value problem-see the examples in Chapter 1 of Protter \& Weinberger (1984); $\tau$ is a priori unknown at all points $z \in$ $(0, l)$, making separate upper and lower solutions, in each subdomain of $[0, l]$, impossible to construct. Restriction (3.11) is of the form $l^{2}<2 /\left(k D_{2} \gamma^{\prime} \bar{C}\right)$, for some constant $k$, suggesting that it is more severe for increasing $D_{2}, \gamma^{\prime}$ and $\bar{C}$.

REMARK 3.2 Violation of Condition (3.11) does not imply that solutions fail to exist, only that (we conjecture) uniqueness may not be assured. This is a possible physical interpretation of the result.

Equations (2.17) constitute a semi-linear elliptic system. Each of the source terms is Lipschitz in its arguments and therefore Hölder continuous with any exponent $\alpha \in(0,1)$. We can prove the existence of at least one solution using the Leray-Schauder fixed-point theorem. We use the variant found in Ladyzhenskaja \& Ural'cera (1968). 
TheOREM 3.1 (Leray-Schauder) Let $\mathcal{M}$ be a Banach space, $t \in[0,1]$ a real parameter, $\mathcal{B}=[0,1] \times \mathcal{M}$ the corresponding space of ordered pairs and $\mathbf{u}$ a real vector. Suppose that $\mathcal{H}(\mathbf{u}, t): \mathcal{B} \rightarrow \mathcal{M}$ is a compact operator continuous in $\mathbf{v} \in \mathcal{M}$ and uniformly continuous in $t$. Suppose further that there exists a constant $M$ such that every possible solution of $\mathcal{H}(\mathbf{u}, t)=\mathbf{0}$ with $\mathbf{u} \in \mathcal{M}$ and $t \in[0,1]$ satisfies $\|\mathbf{u}\|_{\mathcal{B}}<M$. If there exists a unique solution of the equation $\mathcal{H}(\mathbf{u}, 0)=\mathbf{0}$ in $\mathcal{M}$, then there exists a solution of the equation $\mathcal{H}(\mathbf{u}, 1)=\mathbf{0}$ in $\mathcal{M}$.

In order to apply the theorem above, we require that solutions of (2.17) can be contained in a ball in the Banach space $\mathcal{C}[0, l] \equiv C[0, l]^{4}$. Lemma 3.1 provides these bounds.

COROLlary 3.1 (To Lemma 3.1) Classical solutions to (2.17) satisfy the following a priori bound:

$$
|\mathbf{u}|_{\mathcal{C}[0, l]} \equiv\left|X_{2}\right|_{\infty}+\left|X_{1}\right|_{\infty}+|\tau|_{\infty}+|\psi|_{\infty}<M \equiv 1+H+\psi_{\max }+\tau_{\max }
$$

For $t \in[0,1]$, we now define

$$
\mathcal{B}=[0,1] \times \mathcal{M}, \quad \text { where } \mathcal{M} \equiv\left\{\mathbf{u} \in \mathcal{C}[0, l]:|\mathbf{u}|_{\mathcal{C}[0,1]} \leqslant M+\epsilon\right\} .
$$

Here, the bound $M$ is defined as in (3.16) and $\epsilon$ is an arbitrary positive vector. That the bound $M$ holds for all $t \in[0,1]$ is seen by examining (3.1) and the proof of Lemma 3.1. Next, we consider the following family of linear systems:

$$
\begin{gathered}
X_{2}^{\prime \prime}=\frac{\gamma \widehat{X}_{2} \mathrm{e}^{\widehat{\psi} / \widehat{\tau}}}{\gamma+\mathrm{e}^{\widehat{\psi} / \widehat{\tau}}} t-h\left(H \widehat{X}_{1}-\widehat{X}_{2}\right) t, \quad X_{1}^{\prime \prime}=\frac{h}{\eta}\left(H \widehat{X}_{1}-\widehat{X}_{2}\right) t, \\
\psi^{\prime \prime}=-\frac{\kappa \gamma \widehat{X}_{2} \mathrm{e}^{\widehat{\psi} / \widehat{\tau}}}{\gamma+\mathrm{e}^{\widehat{\psi} / \widehat{\tau}}} t, \quad \tau^{\prime \prime}=-(s \widehat{\tau}+\widehat{\psi}) \frac{\Delta \gamma \widehat{X}_{2} \mathrm{e}^{\widehat{\psi} / \widehat{\tau}}}{\gamma+\mathrm{e}^{\widehat{\psi} / \widehat{\tau}}} t, \\
\psi(l)=\bar{\psi} t, \quad \tau(0)=1, \quad X_{2}^{\prime}(l)=X_{1}^{\prime}(l)=0, \\
X_{2}(0)=H X_{1}(0)=t, \quad \psi^{\prime}(0)=\tau^{\prime}(l)=0,
\end{gathered}
$$

where for each $t \in[0,1]$, the system is uniformly elliptic. Problem (3.17) can be written more compactly as

$$
\mathbf{u}(x ; t)^{\prime \prime}=\mathbf{f}_{t}(\mathbf{v}),
$$

where $\mathbf{u}$ is a vector defined as in the previous theorem and $\mathbf{f}$ is defined by the right-hand side of (3.17). For each $\mathbf{v}=\left(\widehat{X}_{1}, \widehat{X}_{2}, \widehat{\psi}, \widehat{\tau}\right) \in \mathcal{M}$, these problems define the transformations $\mathbf{u}(x ; t)=\mathcal{T}_{t} \mathbf{v}, t \in[0,1]$; i.e. to each $(t, \mathbf{v}) \in \mathcal{B},(3.17)$ assigns a unique solution $\mathbf{u}(x ; t) \in \mathcal{C}_{2+\alpha}[0, l]$, for any $\alpha \in(0,1)$. The unique solvability of the linear problem (3.17) in the class $\mathcal{C}^{2}[0, l]$ is assured by the classical results for uniformly elliptic linear BVP, using, e.g. Schauder's theorem. In fact, writing $\mathbf{F}_{t} \mathbf{v}(x)$ as the operator associated with $\mathbf{f}_{t}(\mathbf{v})$, we can obtain the following bound:

$$
|\mathbf{u}(x ; t)|_{\mathcal{C}_{2+\alpha}[0, l]} \leqslant C\left|\mathbf{F}_{t} \mathbf{v}(x)\right|_{\mathcal{C}_{\alpha}[0, l]}
$$

for some positive constant $C$ - see the Schauder estimates in Gilbarg \& Trudinger (2001). Thus, from the Lipschitz property of $\mathbf{f}_{t}, u(x ; t) \in \mathcal{C}_{2+\alpha}[0, l]$ for $\mathbf{v} \in \mathcal{C}_{\alpha}[0, l]$. Note that the dependence of the 
boundary conditions for $\tau$ on the parameter $t$ is selected so that $\tau(x ; t)>0$ for all $t$, ensuring a unique solution $\mathbf{u}(x ; 0)=(0,0,0,1)$. To make precise the correspondence between Problem (2.17) and the transformation $\mathcal{T}_{t}$, note that the solutions (in the space $\mathcal{C}_{2+\alpha}[0, l]$ ) to

$$
\begin{aligned}
\mathbf{u}(x ; t)^{\prime \prime} & =\mathbf{f}(\mathbf{u}(x ; t), t) \\
\psi(l ; t)=\bar{\psi} t, \quad \tau(0 ; t) & =1, \quad X_{2}^{\prime}(l ; t)=X_{1}^{\prime}(l ; t)=0, \\
X_{2}(0 ; t)=H X_{1}(0 ; t) & =t, \quad \psi^{\prime}(0 ; t)=\tau^{\prime}(l ; t)=0
\end{aligned}
$$

are fixed points of the transformation $\mathcal{T}_{t}$. The converse is also true (from $\mathbf{u}(x ; t)=\mathcal{T}_{t} \mathbf{u}(x ; t) \in \mathcal{C}_{2}[0, l]$ and bootstrapping). Therefore, (3.21) is equivalent to the family of fixed points of the transformation $\mathcal{T}_{t}$, and for $t=1$, System $(3.21)$, and therefore $\mathcal{T}_{1} u(x ; 1)=u(x ; 1)$, reduces to $(2.17)$.

In order to demonstrate that $\mathcal{T}_{t}$ is completely continuous, we first note that $\mathcal{T}_{t}$ can be extended to $\mathcal{S}_{t}: \mathcal{C}[0, l] \rightarrow \mathcal{W}_{p}^{2}[0, l]$, as in Gilbarg \& Trudinger (2001) or Ladyzhenskaja \& Ural'cera (1968). Now, let $\mathbf{u}_{1}$ and $\mathbf{u}_{2}$ be two solutions of (3.21) corresponding to $\left(t, \mathbf{v}_{1}\right)$ and $\left(t, \mathbf{v}_{2}\right) \in \mathcal{B}$, respectively. Then, $\mathbf{u}_{1}-\mathbf{u}_{2}$ satisfies

$$
\left\|\left(\mathcal{S}_{1} \mathbf{u}_{1}-\mathcal{S}_{1} \mathbf{u}_{2}\right)^{\prime \prime}\right\|_{\mathcal{W}_{p}^{2}[0, l]} \leqslant\left|\mathbf{f}\left(\mathbf{u}_{1}, t\right)-\mathbf{f}\left(\mathbf{u}_{2}, t\right)\right|_{\mathcal{C}[0, l]}
$$

from the Lipschitz property of $\mathbf{f}$ and its uniform continuity in $t \in[0,1]$. We therefore have continuity of $\mathcal{S}$ in $\mathbf{v} \in \mathcal{C}[0,1]$. From the compactness of the identity $\mathcal{I}: \mathcal{W}_{p}^{2}[0, l] \rightarrow \mathcal{C}[0, l]$, for sufficiently large $p$, we can now extend $\mathcal{T}_{t}$ to an operator $\mathcal{T}_{t}{ }^{*}=\mathcal{S}_{t} \circ \mathcal{I}$ that maps an arbitrary bounded set in $[0,1] \times \mathcal{C}[0, l]$ into a compact set in $\mathcal{C}[0, l]$ and is continuous in $\mathbf{v}$. Uniform continuity of $\mathcal{T}_{t}$ in $t$ follows from the definition of $\mathcal{T}_{t}$.

THEOREM 3.2 (Existence) Let the assumptions of Lemma 3.1 apply. Then there exists a solution $\mathbf{u} \in$ $\mathcal{C}_{2+\alpha}[0, l]$ to BVP $(2.17)$.

Proof. Consider the homotopy $\mathcal{H}_{t} \equiv \mathcal{I}-\mathcal{T}_{t}^{*}$, where $\mathcal{I}$ is the identity map on $\mathcal{C}[0, l]$. This operator inherits the compactness and continuity properties of $\mathcal{T}_{t}^{*}$. It is obvious that $\mathcal{H}_{t} \neq \mathbf{0}$ for any $\mathbf{u} \in \partial \mathcal{M}$. Furthermore, for $t=0$, the unique solution of (3.21) is $\mathbf{u}=(0,0,0,1)$. Therefore, a fixed point exists for $t=1$; or what is the same, the Leray-Schauder degree of the transformation is well-defined and $\operatorname{deg}\left(\mathcal{H}_{1}, \mathcal{M}, \mathbf{0}\right)=\operatorname{deg}\left(\mathcal{H}_{0}, \mathcal{M}, \mathbf{0}\right)=-1$, and thus existence is proved in $\mathcal{C}_{2+\alpha}[0, l]$.

\section{Asymptotic structure of the solutions}

Equations (2.17) are amenable to an asymptotic analysis in appropriate limits. To expose these limits, we estimate the values of the dimensionless quantities in (2.17). These are shown in Table 2. There is a significant degree of uncertainty in a number of influential parameters. Some of the values reported for the quantities appearing in Table 1 differ by several orders of magnitude. $i_{O_{2}, \mathrm{c}}$, e.g. is taken as $4.26 \times 10^{2} \mathrm{~A} \mathrm{~m}^{-2}$ in Berg et al. (2004) but assumes the value $3.85 \times 10^{-4} \mathrm{~A} \mathrm{~m}^{-2}$ in Sun et al. (2005). The main reasons for these discrepancies are

- the difficulties associated with experimental measurement,

- the values are often evaluated by fitting numerical results to experimental data,

- differences in composition and preparation and

- differences in operating conditions. 
TABLE 2 Estimates for the dimensionless parameters in (2.17). $\kappa, \Delta$ and $\eta$ depend on $D_{2}$, so their values can vary by several orders of magnitude

\begin{tabular}{lc}
\hline Quantity & Approximate size \\
\hline$\phi_{\mathrm{c}}$ & $0.03-0.12$ \\
$\kappa / \bar{C}$ & $10^{-6}$ to $10^{-2}$ \\
$h$ & 1 to $10^{-4}$ \\
$\eta$ & 0.1 to $10^{-6}$ \\
$s$ & $20-40$ \\
$\Delta / \bar{C}$ & $10^{-8}$ to $10^{-2}$ \\
\hline
\end{tabular}

This underlines the benefit of an asymptotic analysis; that ranges of parameter space can be explored relatively easily. Moreover, the asymptotic solutions will explicitly demonstrate the dependence of $\mathrm{O}_{2}$ concentration, overpotential and temperature on the dimensionless quantities (2.16), indicating how to gain a measure of control over their values. We split the analysis into three parts, according to the strength of the reaction on the concentration of $\mathrm{O}_{2}$. Since $\gamma$ can be small or large independently of $D_{2}$ (see (2.11)), we can exploit the limit in $\Delta$ and $\kappa$ corresponding to the upper bound of $D_{2}$ in Table 1 (see (2.16)). In this limit, corresponding to weak diffusion limitation (through phase 2), $\Delta \gamma \max \{g, \bar{\psi}\}$ and $\kappa \gamma$ can be $\mathrm{O}(1)$ leading to $\mathrm{O}(1)$ variations in temperature and/or overpotential though we point out that the variations will typically be smaller. Nevertheless, this type of approach to clearly identify trends is commonplace in asymptotic analysis and is known to yield qualitatively accurate information (e.g. the 'high activation energy' limit in combustion theory).

The numerical solutions presented below were generated with the MATLAB BVP solver bvp4c.

\subsection{Strongly $\mathrm{O}_{2}$-dependent reaction: $\gamma \ll \mathrm{e}^{\psi / \tau}$}

We assume firstly that $\ln \gamma \ll \psi / \tau$ ( $\mathrm{e}^{\psi / \tau}$ is large in comparison with $\gamma$ ). This could happen, e.g. if (i) $\gamma \ll 1$ and $\psi>0$ or (ii) if $\mathrm{e}^{\bar{\psi}} \gg \gamma$ and variations in $\psi$ and $\tau$ are asymptotically small (from the values $\bar{\psi}$ and 1 , respectively), both of which are possible. Thus, $\gamma$ need not necessarily be small. In fact, since $\psi_{\mathrm{c}} \ll 1$ for typical values of $\bar{\phi}, \mathrm{e}^{\psi / \tau}$ is generally large, particularly if variations in $\psi$ from $\bar{\psi}$ are small. We further employ the limits $\eta \rightarrow 0, \Delta \rightarrow 0$ and $\kappa \rightarrow 0$, all of which are physically realistic. The analysis proceeds in two parts, depending on the size of $h$.

(1) $h>\mathrm{O}(\eta)$ (fast exchange):

In this limit, the exchange of $\mathrm{O}_{2}$ from phase 1 to phase 2 occurs instantaneously at leading order. With other limits as above, we expand as follows:

$$
X_{2} \sim X_{2,0}, \quad X_{1} \sim X_{1,0}, \quad \psi \sim \psi_{0}, \quad \tau \sim \tau_{0},
$$

yielding

$$
X_{2,0}^{\prime \prime}=\gamma X_{2,0}, \quad H X_{1,0}=X_{2,0},
$$

which has a solution, subject to the boundary conditions (2.17f), given by

$$
X_{2} \sim X_{2,0}=H \frac{\cosh (\sqrt{\gamma}(z-l))}{\cosh (\sqrt{\gamma} l)}, \quad X_{1} \sim X_{1,0}=H^{-1} X_{2,0} .
$$




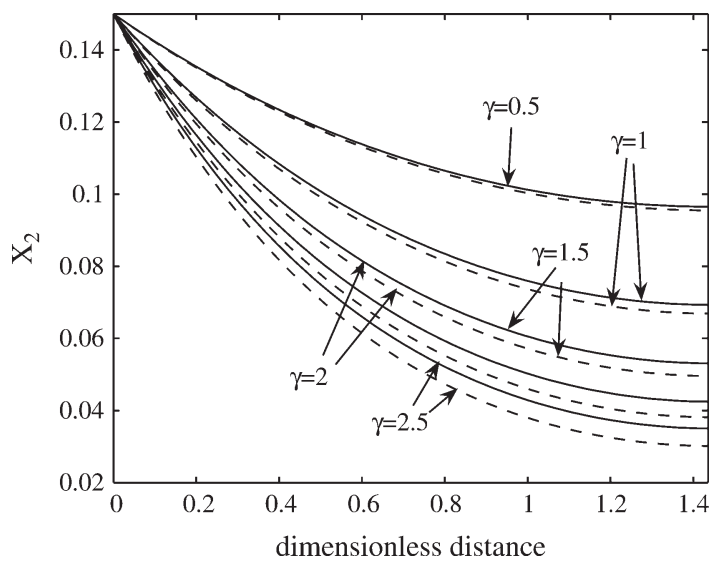

FIG. 4. Comparison of leading-order asymptotic and numerical solutions: plots of $X_{2,0}$, as given by (4.3), against the numerically computed solution of the full system (2.17). In all calculations, $\bar{\psi}=3, \Delta=\kappa=1 / 50$ and $h=125 \eta=1.7 \times 10^{-3}$.

The concentration in the electrolyte reaches a minimum of $H / \cosh (\sqrt{\gamma} l)$ at $z=l$, and approaches the solution $X_{2,0} \equiv H$ as $\gamma \rightarrow 0$. Figure 4 demonstrates that the asymptotic solutions compare very well with the numerical solutions of the full system (2.17). From (2.17e), we find that the current density satisfies

$$
I \sim I_{0} \equiv \gamma \int_{0}^{l} X_{2,0} \mathrm{~d} x=H \sqrt{\gamma} \tanh (\sqrt{\gamma} l), \quad I_{\lim } \sim I
$$

and has the asymptotic behaviour $I \sim \gamma H l$ as $\gamma \rightarrow 0$.

In what follows, we shall assume that $\eta$ is small enough that $H X_{1}=X_{2}$ to first order in all small parameters. An alternative limit is examined later. The leading-order overpotential and temperature problems are then given by

$$
\psi^{\prime \prime} \sim-\kappa \gamma X_{2,0}, \quad \tau^{\prime \prime} \sim-(s \tau+\psi) \Delta \gamma X_{2,0} .
$$

This immediately yields the overpotential profile

$$
\psi-\bar{\psi} \sim \psi_{0}-\bar{\psi}=\psi_{1} \equiv \kappa H \sqrt{\gamma} \tanh (\sqrt{\gamma} l)(l-z)-\kappa H \frac{\cosh (\sqrt{\gamma}(z-l))-1}{\cosh (\sqrt{\gamma} l)},
$$

where $\psi-\bar{\psi} \leqslant \mathrm{O}(1)$. Figure 5 demonstrates that there is excellent agreement between this solution and the numerically computed solution of (2.17). Note further that $\psi$ is monotonically decreasing in $z$, as can be seen by integrating the first of (4.5), and has a maximum value

$$
\psi_{0}(0)=\bar{\psi}+\kappa H l \sqrt{\gamma} \tanh (\sqrt{\gamma} l)-\kappa H \frac{\cosh (\sqrt{\gamma} l)-1}{\cosh (\sqrt{\gamma} l)} .
$$

At this point in the analysis, two distinguished limits exist, based on the size of $\Delta \gamma \max \{s, \bar{\psi}\}$. $\Delta \gamma$ is likely to be small, but we permit $\Delta \gamma \max \{s, \bar{\psi}\}$ and $\kappa \gamma$ to be $\mathrm{O}(1)$ as $D_{2}$ approaches its upper bound in Table 1 . This corresponds to weak diffusion limitation. 

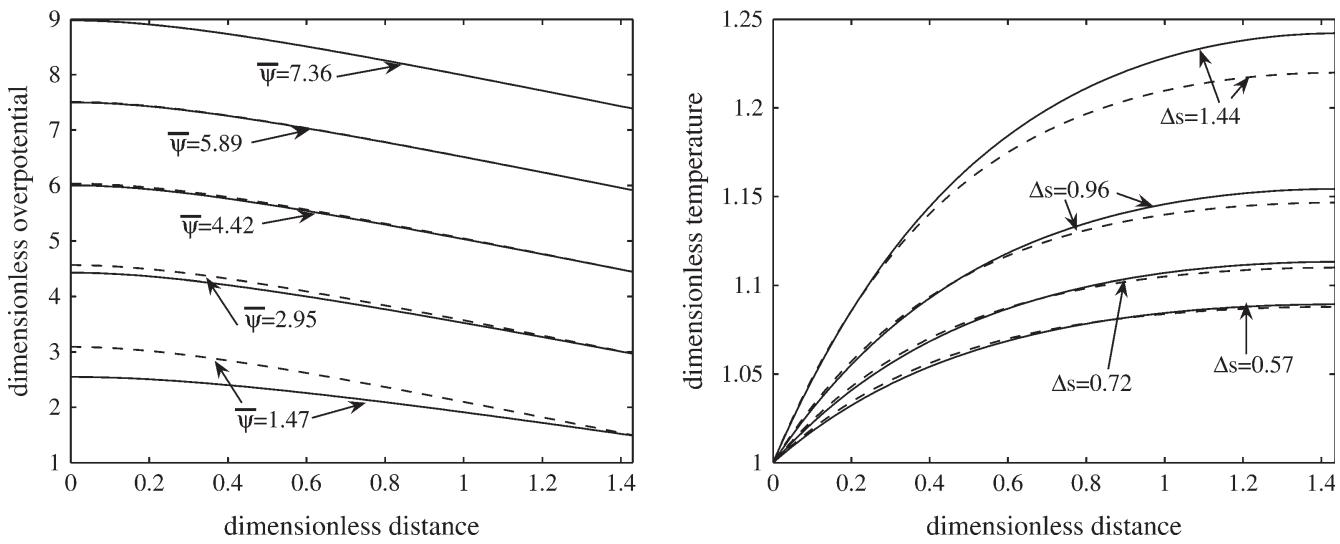

FIG. 5. (Left) A comparison of the numerically computed profiles of $\psi$ (solid lines), according to (2.17) and (2.17f), and the first-order asymptotic solution $\bar{\psi}+\psi_{1}$ given by (4.6) (dashed lines). In these calculations, $h=125 \eta=1.7 \times 10^{-3}, \kappa=3, \gamma=2$ and $\Delta=1 / 40$. (Right) A comparison of the numerically computed profiles of $\tau$ (solid lines), according to (2.17) and (2.17f), and the first-order asymptotic solution given by (4.8) (dashed lines). In these calculations, $\kappa=1 / 40, \gamma=1, h=1.7 \times 10^{-3}=125 \eta$, $\bar{\psi}=3$ and $\Delta$ is varied.

- Strong diffusion limitation:

If $\Delta \gamma \max \{s, \bar{\psi}\} \ll 1$, the rate of $\mathrm{O}_{2}$ diffusion to the reaction sites is slow and variations in temperature from unity are weak, i.e. $\tau_{0}=1$.

Assuming that overpotential variations are small, $\kappa \gamma \ll 1$, by substituting (4.6) into (4.5), we obtain

$$
\tau \sim 1+\Delta(s+\bar{\psi})\left(H-X_{2,0}\right)
$$

Figure 5 compares (4.8) to the numerically computed solution of (2.17).

- Limit of weak diffusion limitation:

Again assuming that $\kappa \gamma \ll 1$, if $\Delta \gamma \max \{s, \bar{\psi}\} \ll 1$, we recover the solutions (4.6). If both $\kappa \gamma=\mathrm{O}(1)$ and $\Delta \gamma s=\mathrm{O}(1)$, variations in $\psi$ and $\tau$ (from $\bar{\psi}$ and unity) are appreciable at leading order. Suppose this to be the case, and for generality, $s / \bar{\psi}=\mathrm{O}(1)$, then we have the following linear problem for $\tau_{0}$ :

$$
\tau_{0}^{\prime \prime}+\Delta \gamma\left(s \tau_{0}+\psi_{0}\right) X_{2,0}=0, \quad \tau_{0}(0)-1=\tau_{0}^{\prime}(l)=0,
$$

where $\psi_{0}$ is given by (4.6). An explicit solution is not possible to find but we can derive useful upper and lower bounds. Using the function $w(z)$ defined in (3.10), the corresponding restriction (3.11) on the size of $l$ and the definition of $X_{2,0}$, it is a relatively simple matter to demonstrate that solutions are bounded below by unity and monotonically increasing in $z$. We can again employ the function $w$ to find sharper upper and lower solutions to (4.9), $\bar{\tau}$ and $\underline{\tau}$, respectively (lower solutions are defined by reversed inequalities in (3.13), with $\tau$ replaced by $\left.\tau_{0}\right)$ :

$$
\bar{\tau}(z)=1+\frac{G\left(1+\psi_{0}(0) / s\right)}{2-G l^{2}} z(2 l-z), \quad \underline{\tau}(z)=1+\frac{G(1+\bar{\psi} / s)}{2 \cosh (\sqrt{\gamma} l)} z(2 l-z),
$$




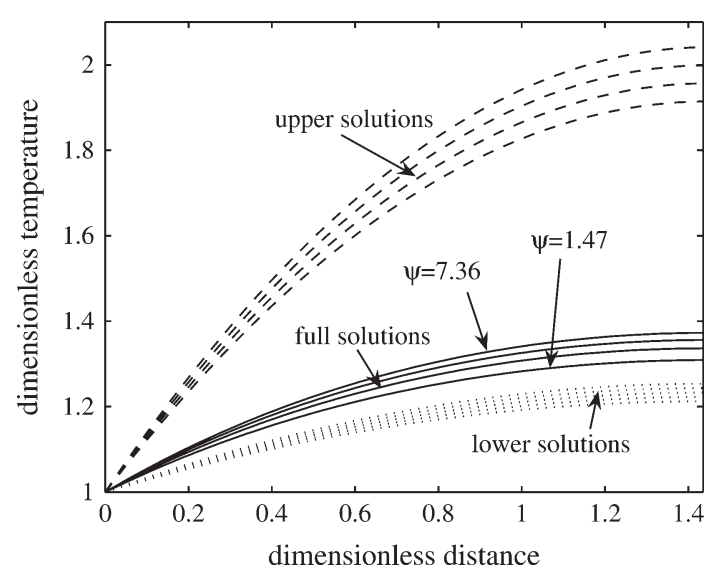

FIG. 6. A comparison of the numerically computed profiles of $\tau$ (solid lines), according to (2.17), and the upper solutions (dasheddotted lines) and lower solutions (dashed lines) given by (4.10). In these calculations, $h=125 \eta=1.7 \times 10^{-3}, \kappa=1 / 20, \gamma=1$ and $\Delta=1 / 10$.

where $G=\Delta \gamma H s$. Note that $\bar{\tau}$ is the same as $\tau_{m}$ defined in (3.15) and $\underline{\tau}$ is an improvement on the previously computed $\underline{\tau}=1$. Importantly, this gives the following range of values that $\tau$ can assume at $z=l$ :

$$
\tau_{0}^{\min } \equiv 1+\frac{G l^{2}}{2 \cosh (\sqrt{\gamma} l)}\left(1+\frac{\bar{\psi}}{s}\right) \leqslant \tau_{0}(l) \leqslant \tau_{0}^{\max } \equiv 1+\frac{G l^{2}}{2-G l^{2}}\left(1+\frac{\psi_{0}(0)}{s}\right) .
$$

Figure 6 demonstrates that (4.10) are indeed upper and lower bounds to the solution of the temperature problem in (2.17). The lower bound actually approximates the solution with good accuracy.

(2) $h=\mathrm{O}(\eta)$ (slow exchange):

In the preceding analysis, we assumed that $h \ll \eta$, yielding solutions of the form (4.2). We now examine the behaviour of the system under the alternative assumption that $h=\mathrm{O}(\eta), \eta \rightarrow 0$, in which case the convective exchange of $\mathrm{O}_{2}$ between the two phases is relatively slow. Considering expansions again of the form (4.1), we obtain, at leading order in the other parameters,

$$
X_{2,0}^{\prime \prime}=\gamma X_{2,0}, \quad X_{1,0}^{\prime \prime}=\mathrm{h}\left(H X_{1,0}-X_{2,0}\right),
$$

where $\mathrm{h}=h / \eta=\mathrm{O}(1)$. These equations are subject to the boundary conditions (2.17f) and the solution is

$$
X_{1,0}=\left(\frac{1}{H}+\Gamma\right) \widehat{X}-\Gamma X_{2,0}, \quad X_{2,0}=H \frac{\cosh (\sqrt{\gamma}(z-l))}{\cosh (\sqrt{\gamma} l)},
$$

where $\Gamma$ and $\widehat{X}$ are defined as

$$
\Gamma=\frac{\mathrm{h}}{\gamma-\mathrm{h} H} \quad \text { and } \quad \widehat{X}=H \frac{\cosh (\sqrt{\mathrm{h} H}(l-z))}{\cosh (\sqrt{\mathrm{h} H} l)} .
$$


The current density is still given at leading order by (4.4). Examining solution (4.13) and (4.14), we see that as $\mathrm{h} \rightarrow \infty, H X_{1,0} \sim X_{2,0}-\gamma X_{2,0} /(\mathrm{h} H)$, which recovers the solution (4.3). In the alternative limit $\mathrm{h} \rightarrow 0$, the solution (4.13) and (4.14) satisfies $X_{1,0}=1+\mathrm{O}(\mathrm{h})$, so the agreement with the exact solution should again be good.

If $\Delta \gamma \max \{s, \bar{\psi}\}=\mathrm{O}(1)$, the bounds (4.10) and (4.11) continue to hold. The mass-exchange term on the right-hand side of (2.17a) will introduce a term of order $h \ll 1$. A simple computation then reveals that corrections to the reaction rate, $\gamma X_{2} \mathrm{e}^{\psi / \tau} /\left(\gamma+\mathrm{e}^{\psi / \tau}\right)$, are of the order of the maximum of $\gamma h, \gamma^{2} \mathrm{e}^{-\psi_{0} / \tau_{0}}, \gamma\left(\psi-\psi_{0}\right)^{2}$ and $\gamma\left(\tau-\tau_{0}\right)^{2}$. Given our assumptions on $\gamma$, the most likely candidate for corrections is $\gamma h$. We therefore expand as follows:

$$
X_{2} \sim X_{2,0}+h X_{2,1}, \quad X_{1} \sim X_{1,0}+h X_{1,1}
$$

retaining terms of the order $\gamma h$ for generality. This yields the following first-order problem:

$$
\begin{gathered}
X_{2,1}^{\prime \prime}-\gamma X_{2,1}=\frac{\gamma}{\mathrm{h}} \Gamma\left(X_{2,0}-\widehat{X}\right), \\
X_{1,1}^{\prime \prime}=\mathrm{h}\left(H X_{1,1}-X_{2,1}\right),
\end{gathered}
$$

subject to the homogeneous form of (2.17f). While these equations have exact solutions, we can gain the information we need simply from maximum principles. The right-hand side of the first equation is positive (negative) if $\widehat{X}<X_{2,0}\left(\widehat{X}>X_{2,0}\right)$, which is ensured if $\widehat{X}(l)<X_{2,0}(l)$ $\left(\widehat{X}(l)>X_{2,0}(l)\right)$, i.e. $\mathrm{h} H>\gamma(\mathrm{h} H<\gamma)$. From the generalized maximum principle and Hopf's lemma, $X_{2,1}$ is then negative (positive). Given $\mathrm{h} H$ and $\gamma$, we can go further. Suppose that $\widehat{X}<$ $X_{2,0}$ and $\max \left(X_{2,0}-\widehat{X}\right)=M\left(\min \left(X_{2,0}-\widehat{X}\right)=0\right)$, then $X_{2,1}$ is bounded below by solutions to

$$
\widetilde{X}_{2,1}^{\prime \prime}-\gamma \widetilde{X}_{2,1}=\frac{\gamma}{\mathrm{h}} \Gamma M, \quad \widetilde{X}_{2,1}(0)=\widetilde{X}_{2,1}^{\prime}(l)=0 .
$$

Though we can find an explicit solution, a bound of the form $a z(2 l-z)$ is easier to interpret. Thus, we obtain the following:

$$
-\frac{\gamma \Gamma / \mathrm{h}}{2+\gamma l^{2}} M z(2 l-z) \leqslant X_{2,1} \leqslant 0 \quad(\mathrm{~h} H>\gamma) .
$$

On the other hand, if $\max \left(X_{2,0}-\widehat{X}\right)=0$ and $\min \left(X_{2,0}-\widehat{X}\right)=-m<0$, then $X_{2,1}$ is bounded as follows:

$$
0 \leqslant X_{2,1} \leqslant \frac{\gamma \Gamma}{2 \mathrm{~h}} m z(2 l-z) \quad(\mathrm{h} H<\gamma)
$$

Expanding in the form $I \sim I_{0}+I_{1}$, where $I_{0}$ is given by (4.4), the correction satisfies

$$
0 \leqslant I_{1} \leqslant \frac{5}{12} \gamma \Gamma m l^{3} \eta(\mathrm{h} H<\gamma), \quad-\frac{5 \gamma \Gamma / 6}{2+\gamma l^{2}} M l^{3} \eta \leqslant I_{1} \leqslant 0(\mathrm{~h} H>\gamma) .
$$

Thus, if the rate of $\mathrm{O}_{2}$ transfer from the phase 2 bulk to the agglomerate surfaces, expressed through $\gamma$, is small in relation to the rate of mass transfer to phase 2 from phase 1 , expressed through $\mathrm{hH}$, the current density is decreased at first order. The converse is also true. 


\subsection{Moderate dependence on $\mathrm{O}_{2}$ concentration: $\gamma=\mathrm{O}\left(\mathrm{e}^{\psi}\right)$}

We move now to the regime in which $\gamma$ is comparable with $\mathrm{e}^{\psi}$, which is certainly the case if $\gamma=\mathrm{O}\left(\mathrm{e}^{\bar{\psi}}\right)$. As with the limit of Section 4.1, we delineate between relatively large and relatively small values of $h$, beginning with instantaneous mass exchange.

(1) $h>\mathrm{O}(\eta)$ (fast exchange):

- Strong diffusion limitation:

Considering expansions of the form (4.1), for weak temperature and overpotential variations, the solution for $\mathrm{O}_{2}$ concentration at leading order is

$$
X_{2,0}=H X_{1,0}=H \frac{\cosh (\sqrt{\underline{\gamma}}(z-l))}{\cosh (\sqrt{\underline{\gamma}} l)}, \quad \underline{\gamma} \equiv \gamma \mathrm{e}^{\bar{\psi}} /\left(\gamma+\mathrm{e}^{\bar{\psi}}\right),
$$

which are the same as the solutions (4.3) of Section 4.1 with $\gamma$ replaced by $\gamma$ (as before, we shall assume that $\eta$ is small enough that $H X_{1}=X_{2}$ to first order in all small parameters). The current density is given at leading order by

$$
I \sim I_{0}=H \sqrt{\underline{\gamma}} \tanh (\sqrt{\underline{\gamma}} l), \quad I_{\lim } \sim I .
$$

- Limit of weak diffusion limitation:

We now assume that only variations in overpotential are small, i.e. $\kappa \ll 1$, and therefore $\psi \sim \bar{\psi}$. Solutions are then given by the problem

$$
X_{2,0}^{\prime \prime}=\gamma \frac{\mathrm{e}^{\bar{\psi} / \tau}}{\gamma+\mathrm{e}^{\bar{\psi} / \tau}} X_{2,0}, \quad X_{1,0}=X_{2,0}
$$

to be solved subject to the boundary conditions (2.17f). $X_{2,0}$ cannot attain a nonnegative maximum and a nonpositive minimum in $(0, l)$, by application of the generalized maximum principle. We can use this fact to determine that $0 \leqslant X_{2,0} \leqslant H$. For strong temperature variations, with $\psi \sim \bar{\psi}$, the solution satisfies

$$
\tau_{0}^{\prime \prime} \geqslant-\bar{G}(\tau+\bar{\psi} / s), \quad \tau_{0}(0)-1=\tau_{0}^{\prime}(l)=0,
$$

having used the upper bound on $X_{2,0}$ and the lower bound of unity for $\tau_{0}$ (the function $\mathrm{e}^{\bar{\psi} / \tau} /\left(\gamma+\mathrm{e}^{\bar{\psi} / \tau}\right)$ is a decreasing function of $\left.\tau\right)$. Here, $\bar{G}=\Delta \gamma H s$, in analogy with the previously defined $G$. Employing the techniques described previously, we can derive the following upper bound:

$$
\tau_{0} \leqslant \bar{\tau}(z)=1+\widetilde{A} z(2 l-z) \leqslant \widetilde{\tau} \equiv 1+\widetilde{A} l^{2}, \quad \widetilde{A} \equiv \frac{1+\bar{\psi} / s}{2-\bar{G} l^{2}} \bar{G} .
$$

We now use this bound to derive sharper estimates for $X_{2,0}$ and $\tau$. Firstly, from (4.22), the monotone decreasing property of $\mathrm{e}^{\bar{\psi} / \tau} /\left(\gamma+\mathrm{e}^{\bar{\psi} / \tau}\right)$ in $\tau$ and the definition (4.24) of $\tilde{\tau}$, we can show that $X_{2,0}$ is bounded above and below by solutions to the equations

$$
\bar{X}_{2,0}^{\prime \prime}=\bar{\gamma} \bar{X}_{2,0}, \quad \underline{X}_{2,0}^{\prime \prime}=\underline{\gamma} \underline{X}_{2,0},
$$


respectively, which have solutions, subject to (2.17f),

$$
\bar{X}_{2,0}=H \frac{\cosh (\sqrt{\bar{\gamma}}(z-l))}{\cosh (\sqrt{\bar{\gamma}} l)}, \quad \underline{X}_{2,0}=H \frac{\cosh (\sqrt{\underline{\gamma}}(z-l))}{\cosh (\sqrt{\underline{\gamma}} l)},
$$

where $\bar{\gamma}=\gamma \mathrm{e}^{\bar{\psi} / \tau} /\left(\gamma+\mathrm{e}^{\bar{\psi} / \widetilde{\tau}}\right)$ and the lower bound corresponds to the solution (4.20). Thus, for weak overpotential variations, increasing temperature variations leads to increasing values of $X_{2}$. A lower bound for $\tau$ is found similarly:

$$
\tau_{0} \geqslant \underline{\tau}(z)=1+\frac{1}{2}\left(1+\frac{\bar{\psi}}{s}\right) \underline{G} z(2 l-z),
$$

where $\underline{G}=\Delta \bar{\gamma} H s / \cosh (\sqrt{\underline{\gamma}} l)$. We use this to bound the current density. Firstly, using the properties of the reaction rate and the lower bound for $X_{2,0}$, we obtain a lower bound

$$
H \bar{\gamma} \tanh (\sqrt{\underline{\gamma}} l) / \sqrt{\underline{\gamma}} \leqslant I_{0} \leqslant H \underline{\gamma} \tanh (\sqrt{\bar{\gamma}} l) / \sqrt{\bar{\gamma}}, \text { yielding } I_{0} \geqslant H \bar{\gamma} \tanh (\sqrt{\underline{\gamma}} l) / \sqrt{\underline{\gamma}},
$$

by the definition of $\bar{\gamma}$ and $\gamma$.

In a similar fashion, if we assume that $\tau \sim 1$ and $\kappa=\mathrm{O}(1)$, then at leading order,

$$
-\psi_{0}^{\prime \prime}=\kappa X_{2,0}^{\prime \prime}=\frac{\kappa \gamma \mathrm{e}^{\psi_{0}}}{\gamma+\mathrm{e}^{\psi_{0}}} X_{2,0} \quad \text { with } 1-\frac{\psi_{0}(l)}{\bar{\psi}}=1-\frac{X_{2,0}(0)}{H}=\psi_{0}^{\prime}(0)=X_{2,0}^{\prime}(l)=0
$$

and $X_{1,0}=X_{2,0}$. An analysis similar to that above yields

$$
\begin{gathered}
H \frac{\cosh \left(\sqrt{\gamma_{m}}(z-l)\right)}{\cosh \left(\sqrt{\gamma}_{m} l\right)} \leqslant X_{2,0} \leqslant H \frac{\cosh (\sqrt{\underline{\gamma}}(z-l))}{\cosh (\sqrt{\underline{\gamma}} l)}, \quad \bar{\psi} \leqslant \psi_{0} \leqslant \bar{\psi}+\frac{\frac{1}{2} H \kappa \gamma\left(l^{2}-z^{2}\right)}{\cosh (\sqrt{\underline{\gamma}} l)}, \\
\psi_{\max } \equiv \bar{\psi}+\frac{1}{2} \frac{H \kappa \gamma l^{2}}{\cosh (\sqrt{\underline{\gamma}} l)}, \quad \gamma_{m}=\frac{\gamma \mathrm{e}^{\psi_{\max }}}{\gamma+\mathrm{e}^{\psi_{\max }}},
\end{gathered}
$$

yielding the following estimates for the current density:

$$
H \underline{\gamma} \tanh \left(\sqrt{\gamma_{m}} l\right) / \sqrt{\gamma_{m}} \leqslant I_{0} \leqslant H \gamma_{m} \tanh (\sqrt{\underline{\gamma}} l) / \sqrt{\underline{\gamma}} .
$$

(2) $h=\mathrm{O}(\eta)$ (slow exchange):

- Strong diffusion limitation:

For $h=\mathrm{O}(\eta), \eta \rightarrow 0$ and weak overpotential variations, the leading-order problem for $\mathrm{O}_{2}$ concentration is

$$
X_{2,0}^{\prime \prime}=\gamma \frac{\mathrm{e}^{\bar{\psi} / \tau}}{\gamma+\mathrm{e}^{\bar{\psi} / \tau}} X_{2,0}, \quad X_{1,0}^{\prime \prime}=\mathrm{h}\left(H X_{1,0}-X_{2,0}\right),
$$

subject to the boundary conditions (2.17f), and where $\mathrm{h}=h / \eta=\mathrm{O}(1)$, as before. For weak temperature variations, the solution is given by

$$
X_{2,0}=H \frac{\cosh (\sqrt{\underline{\gamma}}(z-l))}{\cosh (\sqrt{\underline{\gamma}} l)}, \quad X_{1,0}=\left(\frac{1}{H}+\Gamma^{*}\right) \widehat{X}-\Gamma^{*} X_{2,0},
$$


where

$$
\Gamma^{*}=\frac{\mathrm{h}}{\underline{\gamma}-\mathrm{h} H} \quad \text { and } \quad \widehat{X}=H \frac{\cosh (\sqrt{\mathrm{h} H}(l-z))}{\cosh (\sqrt{\mathrm{h} H} l)},
$$

which is the same as (4.13) and (4.14) with $\gamma$ replaced by $\underline{\gamma}$. Furthermore, the current density is still given by (4.21). Letting

$$
\psi \sim \bar{\psi}+\psi_{1}, \quad \tau \sim 1+\tau_{1}
$$

the first-order problem for temperature is

$$
\tau_{1}^{\prime \prime}=-(s+\bar{\psi}) \Delta \underline{\gamma} X_{2,0}, \quad \tau_{1}(0)=\tau^{\prime}(l)=0 ;
$$

the solution to which is

$$
\tau_{1}=(s+\bar{\psi}) \Delta\left(H-X_{2,0}\right)
$$

By analogy with (4.6), the overpotential correction is given by

$$
\psi_{1}=\kappa H \sqrt{\underline{\gamma}} \tanh (\sqrt{\underline{\gamma}} l)(l-z)-\kappa H \frac{\cosh (\sqrt{\underline{\gamma}}(z-l))-1}{\cosh (\sqrt{\underline{\gamma}} l)},
$$

which has a maximum value

$$
\psi_{1}(0)=\kappa H l \sqrt{\underline{\gamma}} \tanh (\sqrt{\underline{\gamma}} l)-\kappa H \frac{\cosh (\sqrt{\underline{\gamma}} l)-1}{\cosh (\sqrt{\underline{\gamma}} l)} .
$$

\subsection{Tafel limit: $\gamma \gg \mathrm{e}^{\psi}$}

If $\gamma \gg \mathrm{e}^{\psi}$, the reaction rate approaches the Tafel law, introducing extreme sensitivity to changes in overpotential and temperature. This is the form used in the homogeneous models discussed in Section 1.

1) $h>\mathrm{O}(\eta)$ (fast exchange):

- Strong diffusion limitation:

With expansions in the form (4.1), the leading-order problem is defined by the following:

$$
X_{2,0}^{\prime \prime}=X_{2,0} \exp \left(\psi_{0} / \tau_{0}\right), \quad H X_{1,0}=X_{2,0}
$$

subject to the boundary conditions (2.17f). With weak overpotential and temperature variations, $\kappa \ll 1$ and $\Delta \max \{s, \bar{\psi}\} \ll 1$, the solution is

$$
X_{2,0}=H \frac{\cosh \left((z-l) \mathrm{e}^{\bar{\psi} / 2}\right)}{\cosh \left(l \mathrm{e}^{\bar{\psi} / 2}\right)} .
$$

The current density at leading order is given by

$$
I \sim I_{0} \equiv H \mathrm{e}^{\bar{\psi} / 2} \tanh \left(l \mathrm{e}^{\bar{\psi} / 2}\right)
$$


Defining $\psi \sim \bar{\psi}+\psi_{1}$ and $\tau \sim 1+\tau_{1}$, variations in overpotential and temperature now satisfy

$$
\psi_{1}^{\prime \prime}=-\kappa \mathrm{e}^{\bar{\psi}} X_{2,0}, \quad \tau_{1}^{\prime \prime}=-(s+\bar{\psi}) \Delta \mathrm{e}^{\bar{\psi}} X_{2,0}, \quad \psi_{1}(l)=\psi_{1}^{\prime}(0)=\tau_{1}(0)=\tau_{1}^{\prime}(l)=0,
$$

with the solution

$$
\begin{gathered}
\psi_{1}=\kappa H \mathrm{e}^{\bar{\psi} / 2} \tanh \left(l \mathrm{e}^{\bar{\psi} / 2}\right)(l-z)-\kappa\left(X_{2,0}-\frac{H}{\cosh \left(l \mathrm{e}^{\bar{\psi} / 2}\right)}\right), \\
\tau_{1}=\Delta(s+\bar{\psi})\left(H-X_{2,0}\right) .
\end{gathered}
$$

Note that these solutions are the same as (4.6) and (4.8) if $\gamma$ is replaced with $\mathrm{e}^{\bar{\psi}}$.

- Limit of weak diffusion limitation:

For only weak temperature variations, the leading-order problem becomes

$$
X_{2,0}^{\prime \prime}=X_{2,0} \mathrm{e}^{\psi_{0}}, \quad \psi_{0}^{\prime \prime}=-\kappa X_{2,0} \mathrm{e}^{\psi_{0}}, \quad H X_{1,0}=X_{2,0}, \quad \tau_{0}=1,
$$

subject to (2.17f). From the generalized maximum principle, we obtain the bounds $0 \leqslant X_{2,0}$ $\leqslant H, \psi_{0} \geqslant \bar{\psi}$ and $\psi^{\prime} \leqslant 0$ (if solutions exist). The following problem defines an upper bound for the problem in $\psi_{0}$ :

$$
\psi_{\mathrm{u}}^{\prime \prime}=-\kappa H \mathrm{e}^{\psi_{\mathrm{u}}}, \quad \psi_{\mathrm{u}}(l)-\bar{\psi}=0, \quad \psi_{\mathrm{u}}^{\prime}(0)=0 .
$$

The theory of monotone iteration guarantees that a solution to the problem in $\psi_{0}$ exists if $\psi_{\mathrm{u}}$ can be constructed. The proof yields both a bound and an existence (see Theorem 2.3.1 in Sattinger, 1973). Problem (4.45) has no solution for $\kappa H \mathrm{e}^{\bar{\psi}}>\lambda_{1}$ and has two solutions for $\kappa H \mathrm{e}^{\bar{\psi}}<\lambda_{1}$ (one solution for $\kappa H \mathrm{e}^{\bar{\psi}}=\lambda_{1}$ ), where $\lambda_{1}$ is the first eigenvalue of the problem $\psi^{\prime \prime}+\lambda \psi=0, \psi^{\prime}(0)=0, \psi(l)=0$. Let us look for an upper solution in the form $\psi_{\mathrm{u}}=\bar{\psi}+c\left(l^{2}-z^{2}\right) / 2$. A sufficient condition on $c$ is

$$
c \mathrm{e}^{c l^{2} / 2} \geqslant \kappa H \mathrm{e}^{\bar{\psi}}
$$

yielding the upper bound

$$
\psi_{0} \leqslant \bar{\psi}+c l^{2}
$$

2) $h=\mathrm{O}(\eta)$ (slow exchange):

With $h=\mathrm{O}(\eta), \eta \rightarrow 0$, the leading-order problem for the $\mathrm{O}_{2}$ concentrations is

$$
X_{2,0}^{\prime \prime}=\mathrm{e}^{\psi_{0} / \tau} X_{2,0}, \quad X_{1,0}^{\prime \prime}=\mathrm{h}\left(H X_{1,0}-X_{2,0}\right),
$$

subject to the boundary conditions $(2.17 \mathrm{f})$, and where $\mathrm{h}=h / \eta=\mathrm{O}(1)$. For weak temperature and overpotential variations, the solution is given by (4.40) and

$$
X_{1,0}=\left(\frac{1}{H}+\Gamma_{*}\right) \widehat{X}-\Gamma_{*} X_{2,0}, \quad \Gamma_{*}=\frac{\mathrm{h}}{\mathrm{e}^{\bar{\psi}}-\mathrm{h} H} \text { and } \widehat{X}=H \frac{\cosh (\sqrt{\mathrm{h} H}(l-z))}{\cosh (\sqrt{\mathrm{h} H} l)},
$$

which is the same as (4.33) and (4.34) with $\mathrm{e}^{\bar{\psi}}$ in the place of $\underline{\gamma}$. Therefore, the current density is still given at leading order by (4.41). 

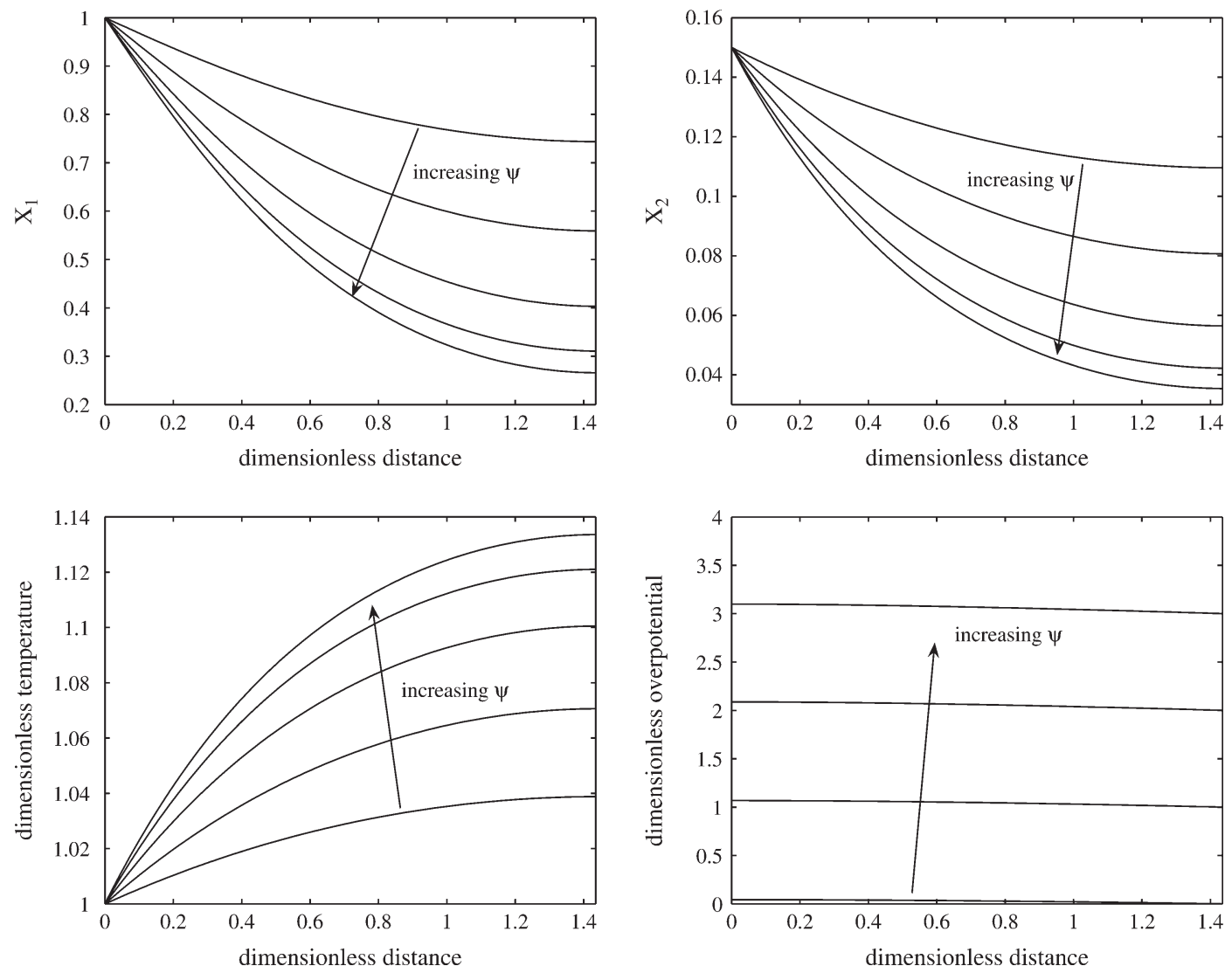

FIG. 7. Typical profiles of $X_{1}, X_{2}, \tau$ and $\psi$, according to (2.17). Profiles correspond to increasing $\bar{\psi}$ in the range $0 \leqslant \bar{\psi} \leqslant 4$ in increments of 1 . For these calculations, $h=125 \eta=1.7 \times 10^{-3}, \gamma=2.5, \Delta=1 / 30$ and $\kappa=1 / 2$.

\section{Numerical results}

In Fig. 7, we show typical profiles of $\mathrm{O}_{2}$ concentration, overpotential and temperature as $\bar{\psi}$ is increased. Here, the variations in overpotential and temperature though not $\mathrm{O}(1)$ are relatively large and are typical of what is seen in laboratory experiments. Figure 8 shows the polarization curves for increasing values of $\gamma$. The limiting current density is reached as $\bar{\psi} \rightarrow \infty$; a consequence of the diffusion limitation on $\mathrm{O}_{2}$, which is modelled through the reaction rate in (2.17). Figure 8 also shows a comparison of the numerically computed dimensionless current density with the composite of the theoretical results (4.4) and (4.21), with $\mathrm{e}^{\bar{\psi}}=3.32$ and $\gamma \leqslant \mathrm{O}\left(\mathrm{e}^{\bar{\psi}}\right)$. For small temperature and overpotential variations, the agreement is excellent, and even for relatively large variations, the errors were found to be small. In the limit of $\gamma \rightarrow \infty$, although not demonstrated in this figure, the agreement was exact.

\section{Discussion and summary}

Modelling of the CCL is far from an easy task, particularly given the difficulty in representing the structure and, moreover, incomplete understanding of some of the physical phenomena. A number of 

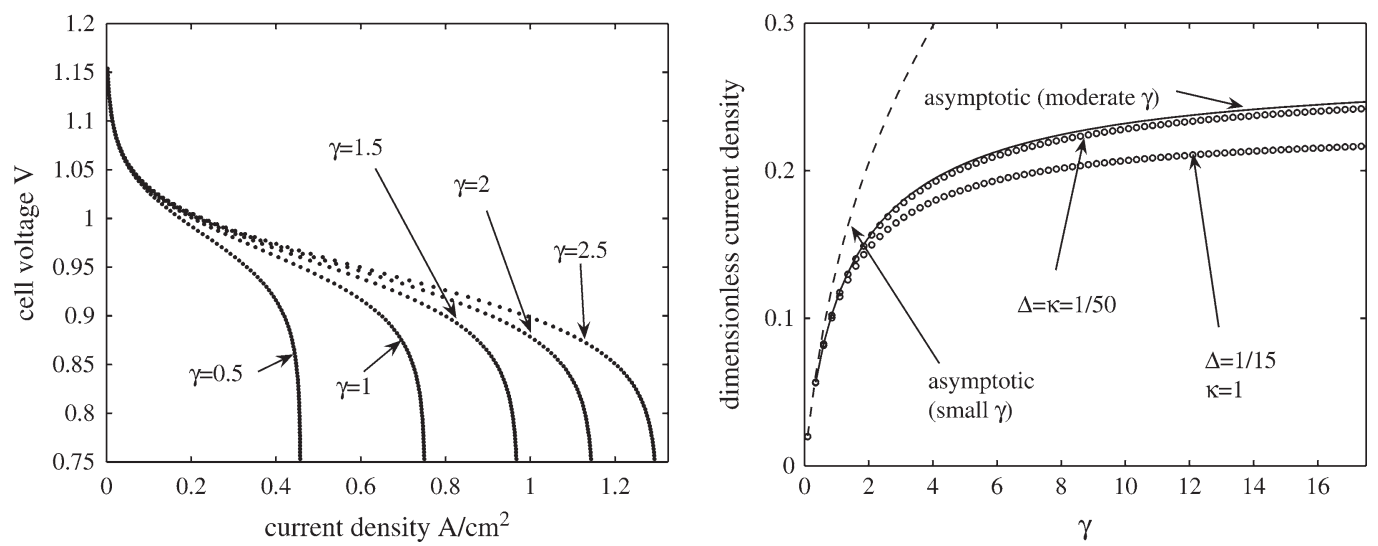

FIG. 8. (Left) Plots of the cell voltage (approximated by $U_{\mathrm{c}}$ ) against the dimensional current density, $I^{\prime}$, as $\gamma$ is increased. In these calculations, $\kappa=\Delta=1 / 30$ and $h=125 \eta=1.7 \times 10^{-3}$. (Right) A comparison of the numerically computed dimensionless current density (hollow circles) with the composite of the theoretical results (4.4) (the dashed line) and (4.21) (the solid line). In these calculations, $\mathrm{e}^{\bar{\psi}}=3.32$ and $\gamma$ covers the ranges $\gamma \ll \mathrm{e}^{\bar{\psi}}$ and $\gamma=\mathrm{O}\left(\mathrm{e}^{\bar{\psi}}\right)$. Two examples are given; one for small temperature and overpotential variations $(\Delta=\kappa=1 / 50)$ and one for large variations $(\Delta=1 / 15, \kappa=1)$. Even for the case of large variations, the agreement is quite good.

the most important processes affecting the performance (and durability), most notably the multiphase nature of the CCL, liquid water transport and heat generation, have proved difficult to include in a mathematical analysis. In this paper, we have accounted for temperature variations and interfacial mass transfer, and analysed the resulting model rigorously. The analysis yields a number of new results, which generally require careful interpretation. We now summarize several of the results that may have the potential to impact on the design of the CCL. For this we recall the definitions of the dimensionless rate coefficient $\gamma$, in (2.16), and the dimensional rate coefficient $\gamma^{\prime}$, in (2.11).

(1) The rate coefficient $\gamma^{\prime}$ can be manipulated by changing the radius of the agglomerates, the volume fraction of electrolyte and the volume fraction of carbon. In practice, this can be achieved by using larger or smaller carbon particles, reducing the ionomer content during preparation, using novel techniques (such as adding charged molecules or applying a magnetic or electrical field, Middelman (2002) and Wang et al. (2005)), using an alternative mixing process or by adding pore formers (Lister \& McLean, 2004).

(2) The dependence of the current density on $\gamma$ is demonstrated in Fig. 8. This dependence, along with the dependence on $l$ and $\bar{\psi}$, can also be seen in the asymptotic expressions (4.4), (4.21) and (4.41), which are either exact or serve as accurate bounds:

(a) When $\gamma \ll \mathrm{e}^{\bar{\psi}}$, the current density increases with increasing $\gamma$ and $l$.

(b) When $\gamma=\mathrm{O}\left(\mathrm{e}^{\bar{\psi}}\right)$ and temperature and overpotential variations are small, the current density increases with increasing $l$ and $\gamma$, defined in (4.20). For larger overpotential and temperature variations, the dependence is more subtle, (4.28), depending also on the temperature solution.

(c) When $\gamma \gg \mathrm{e}^{\bar{\psi}}$, the current density increases with increasing $l$ and $\bar{\psi}$.

(3) The use of Pt across the CCL also depends heavily on the size of $\gamma, \bar{\psi}$ and $l$, through the solutions (4.3), (4.20) and (4.40), which are valid for small leading-order variations in temperature and 
overpotential, for arbitrary $h$, or serve as upper bounds for larger temperature and overpotential variations.

(a) When $\gamma \ll \mathrm{e}^{\bar{\psi}}$, the dependence is entirely on $l$ and $\gamma$. Relatively large values of $\gamma$ and $l$ confine reaction to a region close to the GDL boundary, $z=0$, creating a zone of minimal catalyst utilization (see, e.g. Fig. 4).

(b) When $\gamma=\mathrm{O}\left(\mathrm{e}^{\bar{\psi}}\right)$, reaction is increasingly confined to $z=0$ as $\underline{\gamma}$ and/or $l$ are increased.

(c) When $\gamma \gg \mathrm{e}^{\bar{\psi}}$, reaction becomes more confined as $\bar{\psi}$ and $l$ are increased.

It may appear that decreasing $l, \gamma$ and $\bar{\psi}$ will generally lead to more efficient use of the catalyst. However, decreasing $\gamma$ and $l$ generally decreases the current density, as demonstrated in the previous point. This suggests that the catalyst should be nonuniformly distributed to achieve better performance.

(4) Results (4.7), (4.30), (4.38) and (4.43) provide an indication of the maximum attainable value of the overpotential. Such information could help control unwanted reactions (degradation), such as carbon oxidation and hydrogen peroxide formation (which occur only in certain ranges of cell voltage).

(5) The limiting current density is a good measure of the ideal level of performance of the fuel cell. We point out that high current densities correspond with increased water production, and at high relative humidity, liquid water effects need to be included in the model. Under conditions of low relative humidity assumed in the present work, relation (2.13) demonstrates that the limiting current density is proportional to the quantity $4 F \gamma^{\prime} \bar{C}$, where, from (2.11), $\gamma^{\prime}=4 \pi R_{\text {ag }}^{2} N D_{2} / \delta$.

(6) In general, temperature and overpotential variations will be small, but from the analysis in the limits of weak and strong diffusion limitation, we can readily envisage the trends. For example, results (4.10) and (4.11) demonstrate that the conditions required for an increased current density, as described above, will generally lead to higher temperatures. This is potentially a problem if the fuel cell is operated at low levels of relative humidity, in which case the membrane and electrolyte may dry out, hindering proton migration. However, high current densities also correspond to high levels of water production, though the phase in which it is produced is a matter of some debate. This competition between hydration from water production and dehydration caused by temperature increases is beyond the scope of the present model.

Quite apart from the modelling challenges described above, analysis of a (degenerate) system that includes equations for vapour, dissolved water and liquid water presents a great challenge, even greater if the full dependence on water content and temperature is included. The possible avenues for further investigation are therefore numerous.

\section{Acknowledgements}

The authors would like to thank Ballard Power Systems Inc. and Mathematics of Information Technology and Complex Systems (MITACS) for funding that contributed to this and ongoing work.

\section{REFERENCES}

ARIS, R. (1975) The Theory of the Steady State. The Mathematical Theory of Diffusion and Reaction in Permeable Catalysts, vol. 1. Oxford: Oxford University Press. 
Berg, P., Promislow, K., Pierre, J. S., Stumper, J. \& Wetton, B. (2004) Water management in PeM fuel cells. J. Electrochem. Soc., 151, A341-A353.

CUtLIP, M. (1975) An approximate model for mass transfer with reaction in porous gas diffusion electrodes. Electrochim. Acta., 20, 767-773.

EIKERLing, M. \& Kornyshev, A. (1998) Modelling the performance of the cathode catalyst layer of polymer electrolyte fuel cells. J. Electroanal. Chem., 453, 89-106.

EIKERLING, M. \& Kornyshev, A. (1999) Electrochemical impedance of the cathode catalyst layer in polymer electrolyte fuel cells. J. Electroanal. Chem., 475, 107-123.

Genevey, D. (2004) Transient model of heat, mass and charge transfer as well as electrochemistry in the cathode catalyst layer of a PEMFC. Master's Thesis, Virginia Polytechnic Institute, Blacksburg, VA.

Gilbarg, D. \& Trudinger, N. (2001) Elliptic Partial Differential Equations of Second Order. Springer.

Giner, J. \& HUNTER, C. (1969) The mechanism of operation of the teflon-bonded gas diffusion electrode: a mathematical model. J. Electrochem. Soc., 116, A1124-A1130.

Guo, Q. \& White, R. (2004) A steady-state impedance model for a PEMFC cathode. J. Electrochem. Soc., 151, E133-E149.

HSUEN, H.-K. (2003) Mechanistic approach to performance equations for cathodes in polymer electrolyte fuel cells. J. Power Sources, 123, 26-36.

HuAng, A. \& White, R. (2000) Oxygen diffusion coefficient and solubility in a new proton exchange membrane. J. Electrochem. Soc., 147, A980-A983.

JaOuen, F., Lindbergh, G. \& Sundholm, G. (2002) Investigation of mass-transport limitations in the solid polymer fuel cell cathode: I. Mathematical model. J. Electrochem. Soc., 149, A437-A447.

LAdyzhenskaja, O. \& URAL'CERA, N. (1968) Linear and Quasi-linear Elliptic Equations. New York: Academic Press.

Lin, G., He, W. \& NGuYen, T. V. (2004) Modelling liquid water effects in the gas diffusion and catalyst layers of the cathode of a PEM fuel cell. J. Electrochem. Soc., 151, A1999-A2006.

Lister, S. \& McLean, G. (2004) Review: PEM fuel cell electrodes. J. Power Sources, 130, 61-76.

LIU, F., Yi, B., XING, D., YU, J., HoU, Z. \& FU, Y. (2003) Development of novel self-humidifying composite membranes for fuel cells. J. Power Sources, 124, 81-89.

Mathias, M., Roth, J., Fleming, J. \& Lehnert, W. (2003) Diffusion Media Materials and Characterization, vol. 3, chapter 46. John Wiley.

MAZumder, S. \& Cole, J. (2004) Rigorous 3D mathematical modelling of PEM fuel cells: I. Model predictions without liquid water transport. J. Electrochem. Soc., 150, A1503-A1509.

Middelman, E. (2002) Improved PEM fuel cell electrodes by controlled self-assembly. Fuel Cells Bull., 2002, 9-12.

Mizuhata, H., Shin-IChi, N. \& Yamaguchi, T. (2004) Morphological control of PEMFC electrode by graft polymerization of polymer electrolyte onto platinum-supported carbon black. J. Power Sources, 138, 25-30.

Protter, M. \& Weinberger, H. (1984) Maximum Principles in Differential Equations. New York: Springer.

SAtTInger, D. (1973) Topics in Stability and Bifurcation Theory. Lecture notes in Mathematics, vol. 309. Berlin, Germany: Springer.

Siegel, N., Ellis, M., Nelson, D. \& von Spakovsky, M. (2003) Single domain PEMFC model based on agglomerate catalyst geometry. J. Power Sources, 115, 81-89.

Siegel, N., Ellis, M., Nelson, D. \& Von Spakovsky, M. (2004) A two-dimensional computational model of a PEMFC with liquid water transport. J. Power Sources, 128, 173-184.

Song, D., Wang, Q., LiU, Z., NAvessin, T., Eikerling, M. \& Holdcroft, S. (2004) Numerical optimization study of the catalyst layer of a PEM fuel cell cathode. J. Power Sources, 126, 104-111.

Sun, W., PePpley, B. \& KARAn, K. (2005) An improved two-dimensional agglomerate cathode model to study the influence of catalyst layer structural parameters. Electrochim. Acta, 50, 3359-3374. 
Uchida, M., Aoyama, Y., EdA, N. \& AkiRA, O. (1995) Investigation of the microstructure in the catalyst layer and effects of both perfluorosulfonate ionomer and PTFE-loaded carbon on the catalyst layer of polymer electrolyte fuel cells. J. Electrochem. Soc., 142, A4143-A4149.

Uchida, M., Fukuota, Y., Sugawara, Y., Ohara, H. \& Akira, O. (1998) Improved preparation process of very-low-platinum-loading electrodes for polymer electrolyte fuel cells. J. Electrochem. Soc., 145, A3708-A3713.

UM, S., WANG, C. Y. \& CHEN, K. S. (2000) Computational fluid dynamics modeling of proton exchange membrane fuel cells. J. Electrochem. Soc., 147, A4485-A4493.

WANG, L., WAKAYAMA, N. \& OKADA, T. (2005) Numerical simulation of enhancement of mass transfer in the cathode electrode of a PEM fuel cell by magnet particles deposited in the cathode-side catalyst layer. Chem. Eng. Sci., 60, 4453-4467.

WANG, Q., Eikerling, M., Song, D. \& LiU, Z. (2004) Structure and performance of different types of agglomerates in cathode catalyst layer of PEM fuel cells. J. Electroanal. Chem., 573, 61-79.

Weber, A. \& Newman, J. (2004) Modelling transport in polymer-electrolyte fuel cells. Chem. Rev., 104, 4679-4726.

YIN, K. (2005) Parametric study of proton-exchange membrane fuel-cell cathode using an agglomerate model. J. Electrochem. Soc., 152, A583-A593.

Ziegler, C., Schmitz, A., Tranitz, M., Fontes, E. \& Schumacher, J. (2004) Modeling planar and selfbreathing fuel cells for use in electronic devices. J. Electrochem. Soc., 151, A2028-A2041. 